\title{
Rotating Black Holes in Microscopic Theory: the Implications for Periodic Source M82X-2
}

\author{
Gagik Ter-Kazarian \\ Ambartsumian Byurakan Astrophysical Observatory \\ Byurakan 378433, Aragatsotn District, Armenia \\ Email: gago_50@yahoo.com
}

\begin{abstract}
In previous paper [1], we employ the microscopic theory of black hole to study the observed unusual high luminosity of NuSTAR X-ray pulsations from M82X-2 without the need for significant breaking of Eddington limit. Exploring a spontaneous breaking of gravitation gauge symmetry at huge energies, this theory has smeared out a central singularity of black hole replacing it by the equilibrium, so-called, superdense proto-matter core (SPC), subject to certain rules. In this framework, we think of a M82X-2 as being a spinning intermediate mass black hole. However, for rigorous theoretical solutions, in present report we analytically treat the microscopic model of stationary and axisymmetric rotating black hole. A ring singularity of the Kerr black hole cannot occur, which is now replaced by equilibrium SPC. We calculate the corrections to previous model, introduced by the rotation, of the characteristic phase profile of M82X-2.
\end{abstract}

Keywords: Black hole physics, accretion: accretion discs, X-rays: binaries, X-rays: individual (NuSTAR J095551+6940.8).

\section{Introduction}

In the most striking recent revolutionary NuSTAR discovery [2] of the rare mighty X-ray coherent pulsations coming from the ultraluminous X-ray source M82X-2, located near starburst galaxy M82 (NGC 3034), the source has the maximum luminosity $\widetilde{L}(3-30 \mathrm{keV})=4.9 \times 10^{39} \mathrm{erg} \mathrm{s}^{-1}$, of average period $1.37 \mathrm{~s}$ and a 2.5 -day sinusoidal modulation. This, together with the spin-up behavior, indicative of an accretion torque unambiguously, allows to feature the M82X-2 as mass-exchange binary that contains a nondegenerate secondary donor star.

At first sight, it seems as though the NuSTAR team has demonstrated that the super-Eddington accretion is also possible in ULXs hosting neutron stars, because it is generally believed that the pulsating $\mathrm{X}$-ray sources are magnetic neutron stars which are accreting matter from a binary companion. Therefore, there is nothing left but M82X-2, which until recently astronomers had thought was a black hole, is the brightest magnetic neutron star system ever recorded.

This point of view is widely quoted in literature and, accepted as eminently reasonable. There are, for example, several new results of more recent phenomenological studies (e.g. [3]-[8]) that demonstrate either breaking or circumventing the Eddington limit via somewhat peculiar features of accretion flow onto a highly magnetised neutron stars. However, these approaches are strongly model dependent, and subject to many uncertainties. The physics is obscured by multiple arbitrary assumptions and proliferation of a priori free parameters involved, while a consistent complete theory would not have so many free parameters.

Also, a number of remarks are in orders. The proposals, which are only dependent of geometry of accretion flow, are indeed wholly ruptured when the accretion is ultimately inhibited. This occurs when radiation force is equal to or grater than gravity force, which is just the case of periodic source M82X2. Namely, the pulsed luminosity of M82X-2 reaches about $\sim 26.9$ times brighter than the theoretical threshold at the spherical accretion for $\sim 1.4 M_{\odot}$ stellar-mass black holes where the outward pressure from radiation balances the inward pull of gravity of the pulsar.

This difficulty is brought even into sharper focus by considering the association with M82X-2, which is featured with high luminosity $\left(\simeq 1.8 \times 10^{40} \mathrm{erg} \mathrm{s}^{-1}\right)$ of additional persistent continuous broad X-ray 
radiation observed earlier from its active state [2]. This more compelling argument in somehow or other implies the luminosity $\sim 100$ times if compared to the Eddington limit.

It is equally noteworthy that the centroid of the persistent X-ray emission is between M82X-2 and M82X-1 [2]. If M82X-1 is indeed harbors plausible IMBH, we expect the similarity of the persistent X-ray properties of the M82X-1 and M82X-2 to imply that the non-pulsed emission from the latter originates in the accretion disc, as it must in the black hole M82X-1 [1]. In this sense, the NuSTAR discovery is unexpected and still hard to be explained in the context of magnetic neutron star pulsar model. The only other way of gaining insight into the dynamics of M82X-2 is offered by a black hole.

Instead of making such assumptions of phenomenological studies above, therefore, we find it preferable in previous paper [1] to address the M82X-2 as a spinning intermediate mass black hole (SIMBH) rather than common pulsar, in order to circumvent the obstacles without the need for significant breaking of Eddington limit. It is, of course, foolhardy to contemplate this issue in the framework of Schwarzschild (non-rotating) or Kerr (rotating) black holes. Here and throughout we refer to these models as the phenomenological black hole models (PBHMs), because their only observable integral parameters of total mass and angular momentum still have to put in the theory by hand. The charged black holes are not likely to be important astrophysically. The coherent periodicity of M82X-2 obviously rules out the PBHM, because: (i) Classically, black holes are perfect absorbers but do not emit anything; their physical temperature is absolute zero. (ii) The spinning black holes are axisymmetric and have no internal structure on which to attach a periodic emitter. Orbital motion, whether modulating some emission mechanism directly or exciting short-period pulsations, would decay very quickly due to gravitational radiation.

With this in mind, we have tackled the problem of periodic source of M82X-2 in the framework of microscopic theory of black hole (MTBH) [1]. This theory completes PBHM by exploring the most important processes of spontaneous breaking of gravitation gauge symmetry, and thereof for that of rearrangement of vacuum state at huge energies (subsect. 1.1). In this framework, the M82X-2 is assumed to be SIMBH, resided in final stage of growth.

The thermal blackbody X-ray emission, arising due to the rotational kinetic energy of black hole, escapes from event horizon through the vista to outside world, which is detected as ultraluminous X-ray pulsations. The M82X-2 indeed releases $\sim 99.6 \%$ of its pulsed radiative energy predominantly in the Xray bandpass $0.3-30 \mathrm{keV}$. We derived a pulse profile and gave a quantitative account of energetics and orbital parameters of the semi-detached X-ray binary containing a primary accretor M82X-2 of inferred mass $M \simeq 138.5-226 M_{\odot}$ and secondary massive, $M_{2}>48.3-64.9 M_{\odot}, \mathrm{O} / \mathrm{B}$-type donor star with radius of $R>22.1-25.7 R_{\odot}$, respectively. We computed the torque added to M82X-2 per unit mass of accreted matter which yields the measured spin-up rate.

For brevity reasons in [1], we have refrained from providing rigorous theoretical evolutionary paths of the equations describing the rotating black holes, instead we have proceeded in relatively simple way of considering non-rotating black holes, which is quick to estimate the most important conceptual aspects of associated physics, without loss of generality. However, we need to be more rigorous about a geometry which describes rotating axisymmetric black holes. To fill the void, in present article we analytically study the microscopic model of stationary and axisymmetric rotating black hole. In particular, we derive field equations and obtain both internal and global vacuum spacetime solutions.

We will proceed according to the following structure. To get started, we briefly outline the preceding developments of MTBH in subsection 1.1. We provide an analysis aimed at clarifying the current situation. Section 2 deals with a detailed analytical treatment of basic axisymmetric spacetime geometry, which describes the rotating black holes. The corresponding field equations of stationary and axisymmetric rotating black holes are derived in section 3 . We obtain a global vacuum spacetime solutions and describe the horizons in section 4 . Section 5 presents the microscopic model of rotating SPC, where an assessment of a distinction from Kerr model is given too. The model building of the periodic ULX M82X-2 is brought in section 6 . The concluding remarks are given in section 7 . To make the paper understandable, the specific issues dealt with in the appendices A-F are further details on the underlying gravitation theory, which are in use in previous sections. Unless otherwise stated, we take geometrized units throughout this paper. 


\subsection{The Preceding Developments of MTBH}

Even though being among the most significant advances in astrophysics, it is rather surprising that PBHM is routinely used to explore the growth and merging behavior of black holes. It cannot be accepted as a convincing model for addressing this problem because in PBHM-framework the very source of gravitational field of the black hole is a kind of meaningless curvature singularity at the center of the stationary black hole, which is hidden behind the event horizon. The theory breaks down inside the event horizon which is causally disconnected from the exterior world. Either the Kruskal continuation of the Schwarzschild metric, or the Kerr metric, shows that the static observers fail to exist inside the horizon. Recall that the Kruskal manifold is the maximal analytic extension of the Kerr solutions inside event horizon, so no more regions can be found by analytic continuation.

PMBH then presents a major challenge that renders time reversibility impossible. Objects thrown into the black hole can never be retrieved, because it will get into infinite collapse to a ring singularity inside the black hole. Any object that collapses to form a black hole will go on to collapse to a singularity inside the black hole. Any timelike worldline must strike the central singularity which wholly absorbs the infalling matter. Therefore, the ultimate fate of collapsing matter once it has crossed the black hole surface is unknown.

So, one should deliberately forebear from presumption of exotic hypothetical growth behavior of black holes, which seems nowhere near true if one applies the phenomenological model, which ultimately disables any accumulation of matter in the central part and, thus, neither the growth of black holes nor the increase of their mass-energy density could occur at accretion of outside matter, or by means of merger processes. The PBHM is a rather restricted model and one needs to realise that if one can gain insight into exploring a new process of spontaneous breaking of gravitation at huge energies, one has then made room for growth and merging behavior of black holes. Therefore, a new conceptual framework will be required in order to have a proper understanding of the black hole physics.

Being an extension of PMBH, suitable for applications in ultra-high energy (UHE) astrophysics, the MTBH is a bold assumption in its own right. In this framework, a substantial change of the properties of spacetime continuum, so-called inner distortion (ID), besides the curvature, arises at spontaneous breaking of gravitation gauge symmetry. The matter found in the ID-region of spacetime has undergone phase transition of the second order. In the resulting, so-called proto-matter, the pressure becomes dominant over gravitational force at very short distances when matter falls into central singularity as the collapse proceeds and, thus, it halts the infinite collapse ([9]-[12]).

Therefore, one of the most remarkable drawback of MTBH is that the central singularity cannot occur, which is replaced by finite though unbelievably extreme conditions held in the stable SPC where, nevertheless, static observers exist. The stable SPC, in fact, is the end product of the evolution of massive objects. This makes room for growth and merging properties of black holes, which may shed considerable light upon the growth and merging phenomena of astrophysical black holes, which are in evidence throughout the Universe.

This also ultimately circumvent the principle problem of an observer's inability to access the degrees of freedom that are hidden beyond the horizon, and a necessity to assign the misleading entropy to black hole. The physical entropy of SPC is a measure of the large number of the real thermodynamical microstates, which are compatible with the ergodicity. The SPC is always found inside the event horizon, therefore it could be observed only in presence of accreting matter. The SPC, surrounded by the outside accretion disk, presents the microscopic model of AGN.

The SPC accommodates the highest energy scale up to hundreds $\mathrm{ZeV}$, which accounts for the spectral distribution of the resulting radiation of AGNs. The SPC stood the tests of rigorous theoretical scrutinies of stability. A numerical integration of the stability equations clearly proves the stability of SPC [13]. An external physics of accretion onto the SPC in the first half of its lifetime is identical to the processes in Schwarzschild's model. A crucial difference comes in when one looks for the growth and merging behavior of black holes. In the framework of MTBH, the black hole seeds might grow driven by the accretion of outside matter $[11,12]$.

Understanding how seed black holes grow into IMBHs and supermassive black holes (SMBHs), has important implications for the duty-cycle of AGN, galaxy evolution, and gravitational wave astronomy. Therefore, we have undertaken a large series of numerical simulations with the goal to trace an evolution 
of the mass assembly history of 377 accreting SMBH seeds in AGNs to the present time and examine the observable signatures today.

We also study the emission of UHE-neutrinos with energies up to hundreds ZeV. Such neutrinos are produced by the cooling of the SPC via simple or nucleon-modified URCA processes, and the pionic reactions. As a metric singularity inevitably disappears, the $\mathrm{ZeV}$-neutrinos may escape from event horizon to outside world through a thin belt area, even after the strong neutrino trapping. While hard to detect, the extragalactic ZeV-neutrinos may reveal clues on the puzzle of the origin of ultra-high energy cosmic rays, as they have the advantage of representing unique fingerprints of hadron interactions and, therefore, can initiate via very complex chains of Z-burst interactions the cascades of UHE cosmic rays with energies exceeding $1.0 \times 10^{20} \mathrm{eV}($ e.g. [14]-[16]).

\section{Basic Axisymmetric 4D Spacetime Geometry}

The non-spinning SPC is static and spherically symmetric. Therefore, we need to be clear about more general geometry which can describe rotating axisymmetric SPC. The principle foundation of the spinning configurations first comprises the following additional distinctive features with respect to non-spinning ones: (i) Rapid rotation causes the shape of the configuration to be flattened by centrifugal forcesflattened at poles and bulged at equator (oblate spheroid, which is second order effect in the rotation rate). (ii) A rotating massive configuration drags space and time around with it (non-Newtonian gravitational effect). The local inertial frames are dragged by the rotation of the gravitational field, i.e. a gyroscope orbiting near the configuration will be dragged along with the rapidly rotating configuration. This is probably the most remarkable feature that could serve as a link with the general description of spacetime (also see [17]). Beside the geodetic procession, a spin of the body produces in addition the Lense-Thirring procession.

In the appendices we necessarily recount some of the highlights behind of gravitation theory underlying MTBH, which are in use throughout the sections 2 and 3. The proposed theory explores a spontaneous breaking of gravitation gauge symmetry $([18,9,10]$ and earlier references cited therein, which thoroughly discussed in $[11,12]$ ). Much use has been made of the language of fundamental geometric structure, that is, a distortion gauge induced fiber-bundle, incorporating with the spacetime deformation/distortionframework $[12,19,20]$.

\subsection{Axisymmetric Distortion of $M_{6} \rightarrow V_{6}:$ A Reduction to $V_{4}$}

Before attempting to build an axisymmetric distorted Riemannian $4 \mathrm{D}$ space $V_{4}$, according to proposed gravitation theory (see appendices A-F for details), we need to consider an axisymmetric distortion of the $6 \mathrm{D}$ space $M_{6} \rightarrow V_{6}$, and next reduce it to $V_{4}$. The element $D(a, \theta)$ of the distortion group $G_{D}$ (55) has induced a general distortion transformations (53) and (54) of the six-basis vectors $e=O \times \sigma(\in$ $\left.M_{6}\right) \rightarrow \widetilde{e}=\widetilde{O} \times \widetilde{\sigma}\left(\in V_{6}\right):$

$$
\begin{aligned}
& 2 \widetilde{e}_{(0 \alpha)}=\widetilde{\xi}_{(0 \alpha)} \times\left(\widetilde{\sigma}_{(+\alpha)}+\widetilde{\sigma}_{(-\alpha)}\right)+\widetilde{\xi}_{(\alpha)} \times\left(\widetilde{\sigma}_{(+\alpha)}-\widetilde{\sigma}_{(-\alpha)}\right), \\
& 2 \widetilde{e}_{(\alpha)}=\widetilde{\xi}_{(\alpha)} \times\left(\widetilde{\sigma}_{(+\alpha)}+\widetilde{\sigma}_{(-\alpha)}\right)+\widetilde{\xi}_{(0 \alpha)} \times\left(\widetilde{\sigma}_{(+\alpha)}-\widetilde{\sigma}_{(-\alpha)}\right),
\end{aligned}
$$

where distortion transformations of the ingredient basis vectors $(O)$ and $(\sigma)$ are not independent $(56)$. Hence

$$
\begin{aligned}
& \delta \widetilde{\xi}_{(0 \alpha)}=-\xi_{0} x_{(0 \alpha)}+\xi x_{\alpha}+\bar{\xi}_{0} \bar{x}_{(0 \alpha)}-\bar{\xi} \bar{x}_{\alpha}, \\
& \delta \widetilde{\xi}_{\alpha}=\xi x_{(0 \alpha)}-\xi_{0} x_{\alpha}+\bar{\xi}_{0} \bar{x}_{\alpha}-\bar{\xi} \bar{x}_{0 \alpha)}
\end{aligned}
$$

provided $\delta \widetilde{\sigma}_{A}(\theta)=\delta \mathcal{R}_{A}^{\beta}(\theta) \sigma_{\beta}$.

Let $\phi\left(=x^{1}\right)$ be the azimuthal angle about the axis of symmetry in $R^{3}$ (51). The axisymmetric distortion of the space $M_{6} \rightarrow V_{6}$ can be induced by the distortion gauge field $a_{A}$ (see (71), (72)), with the only non-zero $1 \mathrm{D}$ components of $x_{0}:=x_{(03)}=\kappa a_{( \pm 3)}$ and $x_{1}=\mp \kappa a_{( \pm 1)}$, in presence of ID-field, $\bar{x}:=\bar{x}_{(03)}=\kappa \bar{a}_{( \pm 3)}$, where a coupling constant $\kappa$ relates to the Newton's gravitational constant $G_{N}$ 
by (70). Then, the non-zero components of the transformation matrix $D(44)$ can be recast into the form

$$
\begin{aligned}
& D_{(\tilde{01})}^{(01)}=1, \quad D_{(\tilde{01})}^{1}=x_{1}, \quad D_{(\tilde{02})}^{(02)}=\cos \theta_{1}, \quad D_{(\tilde{02})}^{3}=\sin \theta_{1}, \quad D_{(\tilde{03})}^{(03)}=\cos \theta_{1}\left(1-x_{0}\right), \\
& D_{(\tilde{03})}^{2}=-\sin \theta_{1}\left(1+x_{0}\right), \quad D_{(\tilde{0})}^{\overline{3}}=-\cos \theta_{1}, \quad D_{(\tilde{03})}^{(0 \overline{3})}=-\sin \theta_{1}, \quad D_{\tilde{1}}^{1}=1, \quad D_{\tilde{1}}^{(01)}=-x_{1}, \\
& D_{\tilde{2}}^{2}=\cos \theta_{1}, \quad D_{\tilde{2}}^{(03)}=\sin \theta_{1}, \quad D_{\tilde{3}}^{3}=\cos \theta_{1}\left(1+x_{0}\right), \quad D_{\tilde{3}}^{(02)}=-\sin \theta_{1}\left(1-x_{0}\right), \\
& D_{\tilde{3}}^{(03)}=\cos \theta_{1} x_{1}, \quad D_{\tilde{3}}^{2}=-\sin \theta_{1} x_{1},
\end{aligned}
$$

where the rotation angles are determined as

$$
\pm \theta_{(1)}:=\tan \theta_{( \pm 1)}=\mp x_{1}, \quad \tan \theta_{( \pm 3)}=x_{(0)}, \quad \theta_{(+3)}=\theta_{(-3)} .
$$

The (1) then becomes

$$
\begin{aligned}
& \widetilde{e}_{(01)}=e_{(01)}+e_{1} x_{1}, \quad \widetilde{e}_{(02)}=e_{(02)} \cos \theta_{1}+e_{3} \sin \theta_{1}, \\
& \widetilde{e}_{(03)}=\cos \theta_{1}\left[e_{(03)}\left(1-x_{0}\right)-\bar{e}_{3} \bar{x}\right]-\sin \theta_{1}\left[e_{(2)}\left(1+x_{0}\right)+\bar{e}_{(02)} \bar{x}\right], \\
& \widetilde{e}_{1}=e_{1}-e_{(01)} x_{1}, \widetilde{e}_{2}=e_{2} \cos \theta_{1}+e_{(03)} \sin \theta_{1}, \\
& \widetilde{e}_{3}=\cos \theta_{1}\left[e_{3}\left(1+x_{0}\right)+\bar{e}_{(03)} \bar{x}\right]-\sin \theta_{1}\left[e_{(02)}\left(1-x_{0}\right)-\bar{e}_{2} \bar{x}\right] .
\end{aligned}
$$

Consequently, the resulting deformed metric on $V_{6}$ in holonomic coordinate basis takes the form

$$
\begin{aligned}
& g_{00}=\tau_{1}^{2}\left(1-\tan ^{2} \theta_{1}\right)+\tau_{2}^{2} \cos 2 \theta_{1}+\tau_{3}^{2}\left\{\cos ^{2} \theta_{1}\left[\left(1-x_{0}\right)^{2}+\bar{x}^{2}\right]-\sin ^{2} \theta_{1}\left[\left(1+x_{0}\right)^{2}+\bar{x}^{2}\right]\right\}, \\
& g_{01}=-2 \tau_{1} x_{1}, \quad g_{02}=2 \tau_{3} \sin 2 \theta_{1}, \quad g_{03}=-2 \tau_{2} \sin 2 \theta_{1}, \quad g_{11}=-\left(1+\tan ^{2} \theta_{1}\right), \\
& g_{22}=-\cos 2 \theta_{1}, \quad g_{33}=-\cos ^{2} \theta_{1}\left[\left(1+x_{0}\right)^{2}+\bar{x}^{2}\right]+\sin ^{2} \theta_{1}\left[\left(1-x_{0}\right)^{2}+\bar{x}^{2}\right],
\end{aligned}
$$

where, according to (51), the $3 \mathrm{D}$ space $T^{3}$ is spanned by the coordinates $d \widetilde{x}^{(0 \alpha)}=\tau_{\alpha} d \widetilde{x}^{0} \quad\left(\tau_{1}^{2}+\tau_{2}^{2}+\tau_{3}^{2}=\right.$ 1).

When reducing $V_{6} \rightarrow V_{4}$, we may further fix the most convenient universal time direction by imposing the constraint $g_{02} \widetilde{d x^{2}}+g_{03} d \widetilde{x}^{3}=0$, i.e.,

$$
\sin 2 \theta_{1}\left(\tau_{3} d \widetilde{x}^{2}-\tau_{2} d \widetilde{x}^{3}\right)=0 .
$$

(i) In the case of static spherical-symmetry when $\sin 2 \theta_{1}=0$, the $\tau_{\alpha}$ can be chosen as $\tau_{1}=\tau_{2}=$ $0, \tau_{3}=1[11,12]$.

(ii) Let, in axisymmetric space $V_{4}$, the world coordinate $\widetilde{t}\left(=\widetilde{x}^{0}\right)$ be the time (in units of c), and $\widetilde{\phi}\left(=\widetilde{x}^{1}\right)$ be the azimuthal angle about the axis of symmetry. The space $V_{4}$ would be invariant against simultaneous inversion of time $\widetilde{t}$ and azimuthal angle $\widetilde{\phi}$. So, $\sin 2 \theta_{1} \neq 0$, and the $\tau_{2}$ and $\tau_{3}$ can be chosen to eliminate the term $\left(\tau_{3} d \widetilde{x}^{2}-\tau_{2} d \widetilde{x}^{3}\right)$, which has the effect of introducing the following values into the problem:

$$
\tau_{2}=\tau_{3} \frac{\widetilde{u}^{2}}{u^{3}}, \quad \text { at } \quad \frac{\widetilde{u}^{2}}{\widetilde{u}^{3}} \leq 1, \quad \tau_{3}=\tau_{2} \frac{\widetilde{u}^{3}}{u^{2}}, \quad \text { at } \quad \frac{\widetilde{u}^{3}}{\widetilde{u}^{2}} \leq 1
$$

A velocity field at each point in $V_{4}$ is $\widetilde{u}^{t}=d \widetilde{t} / d \widetilde{s}$, with proper time $d \widetilde{s}, \widetilde{t}\left(=x^{0}\right)$ is the world time coordinate (in units of c), $\widetilde{u}^{\phi}=d \widetilde{\phi} / d \widetilde{s}=\Omega \widetilde{u}^{t}$ and $\widetilde{u}^{t}=d \widetilde{x}^{C} / d \widetilde{s}=\widetilde{u}^{t} \widetilde{v}^{C}$, and $\Omega=d \widetilde{\phi} / \tilde{d}$ is the angular velocity, as seen by an inertial observer at rest at infinity. The components $\widetilde{v}^{C}=d \widetilde{x}^{C} / \widetilde{d t}(C=2,3)$ denote the poloidal velocity.

Hence (6) becomes metric on axisymmetric space $V_{4}$ :

$$
\begin{aligned}
& g_{00}=\tau_{1}^{2}\left(1-\tan ^{2} \theta_{1}\right)+\tau_{2}^{2} \cos 2 \theta_{1}+\tau_{3}^{2}\left\{\cos ^{2} \theta_{1}\left[\left(1-x_{0}\right)^{2}+\bar{x}^{2}\right]-\sin ^{2} \theta_{1}\left[\left(1+x_{0}\right)^{2}+\bar{x}^{2}\right]\right\}, \\
& g_{01}=-2 \tau_{1} x_{1}, \quad g_{11}=-\left(1+\tan ^{2} \theta_{1}\right), \quad g_{22}=-\cos 2 \theta_{1}, \\
& g_{33}=-\cos ^{2} \theta_{1}\left[\left(1+x_{0}\right)^{2}+\bar{x}^{2}\right]+\sin ^{2} \theta_{1}\left[\left(1-x_{0}\right)^{2}+\bar{x}^{2}\right] .
\end{aligned}
$$

We define the five quantities $\nu, \psi, \omega, \mu_{2}$ and $\mu_{3}$ which are only functions of the coordinate $\widetilde{x}^{2}$ and $\widetilde{x}^{3}$ :

$$
\begin{aligned}
& \exp (2 \psi)\left(\widetilde{x}^{2}, \widetilde{x}^{3}\right):=1+x_{1}^{2}, \quad \omega\left(\widetilde{x}^{2}, \widetilde{x}^{3}\right):=-\frac{\tau_{1} x_{1}}{1+x_{1}^{2}}, \quad \exp \left(2 \mu_{2}\right)\left(\widetilde{x}^{2}, \widetilde{x}^{3}\right):=\frac{1-x_{1}^{2}}{1+x_{1}^{2}}, \\
& \exp (2 \nu)\left(\widetilde{x}^{2}, \widetilde{x}^{3}\right):=\frac{1}{1+x_{1}^{2}}\left\{\tau_{1}^{2}\left(1+x_{1}^{2}-x_{1}^{4}\right)+\tau_{2}^{2}\left(1-x_{1}^{2}\right)+\tau_{3}^{2}\left[\left(1-x_{0}\right)^{2}+\bar{x}^{2}\right]-\tau_{3}^{2} x_{1}^{2}\left[\left(1+x_{0}\right)^{2}+\bar{x}^{2}\right]\right\}, \\
& \exp \left(2 \mu_{3}\right)\left(\widetilde{x}^{2}, \widetilde{x}^{3}\right):=\frac{1}{1+x_{1}^{2}}\left\{\left(1+x_{0}\right)^{2}+\bar{x}^{2}-x_{1}^{2}\left[\left(1-x_{0}\right)^{2}+\bar{x}^{2}\right]\right\},
\end{aligned}
$$


where we seek a holonomic coordinates $\widetilde{x}^{\mu}(x)$, in the construction of (45), as the solutions of the first-order partial differential equations:

$$
\frac{\partial \widetilde{x}^{\mu}}{\partial x^{l}} \equiv \psi_{l}^{\mu}=D_{l}^{\mu}(1+\varpi(F)) .
$$

Provided, a world-deformation tensor (Appendix B) is in the form $\Omega_{l}^{m}(F)=\delta_{l}^{m}(1+\varpi(F))$, then $\varpi(F)$ is the scalar function of antisymmetric tensor of the gauge field. We do not at the moment specify the scalar function $\varpi(F)$ apart from the initial condition $\varpi(0)=0$, which can be determined in the intermediate stage of the analysis.

Substituting (10) into a bilinear form of norm $d \widetilde{s}^{2}$ of infinitesimal displacement on $V_{4}$, describing the stationary and axisymmetric spacetimes, we obtain

$$
d \widetilde{s}^{2}=\exp (2 \nu) d \widetilde{t}^{2}-\exp (2 \psi)(d \widetilde{\phi}-\omega d \widetilde{t})^{2}-\exp \left(2 \mu_{2}\right)\left(d \widetilde{x}^{2}\right)^{2}-\exp \left(2 \mu_{3}\right)\left(d \widetilde{x}^{3}\right)^{2}
$$

Thus, we arrive at the metric (12) of the stationary and axisymmetric space $V_{4}$ in the most commonly used $3+1$ formalism (e.g. [21]-[26]).

\subsection{Axisymmetric Space $V_{4}$ in $3+1$ Formalism}

In the $3+1$ formalism, as usual, $3+1$ foliations of spacetime $V_{4}$ by space-like 3 -slices $\left\{\Sigma_{t}\right\}$ play an important role. The study of a dragging effect is assisted by incorporating with the soldering tools in order to relate local Lorentz symmetry to curved spacetime. These are the linear frames and forms in tangent fiber-bundles to the external general smooth differential manifold, whose components are so-called tetrad (vierbein) fields.

That is, the $V_{4}$ has at each point a tangent space, $T_{x} V_{4}$, spanned by the anholonomic orthonormal frame field, $e$, as a shorthand for the collection of the 4-tuplet $e_{0}=\exp (-\nu)\left(\widetilde{\partial}_{t}+\omega \widetilde{\partial}_{\phi}\right), e_{1}=$ $\left.\exp (-\psi) \widetilde{\partial}_{\phi}, e_{2}=\exp \left(-\mu_{2}\right) \widetilde{\partial}_{2}, e_{3}=\exp \left(-\mu_{3}\right) \widetilde{\partial}_{3}\right)$, where $e_{a}=e_{a}{ }^{\mu} \widetilde{\partial}_{\mu}, e_{a}{ }^{\mu}$ is the soldering form between the tangent space and the spacetime manifold. This is called a Bardeen observer, locally nonrotating observer, or the local Zero Angular-Momentum Observers (ZAMO), i.e. observers whose worldlines are normal to the hypersurfaces defined by constant coordinate time, $\widetilde{t}=$ const, also called Eulerian observers. Here we use Greek alphabet $(\mu, \nu, \rho, \ldots=0,1,2,3)$ to denote the holonomic world indices related to $V_{4}$, and the first half of Latin alphabet $(a, b, c, \ldots=0,1,2,3)$ to denote the anholonomic indices related to the tangent space.

The frame field, $e$, then defines a dual vector, $\vartheta$, of differential forms, $\vartheta=\left(\begin{array}{c}\vartheta^{0}=\exp \nu d \widetilde{t} \\ \vartheta^{1}=\exp \psi(d \psi-\omega d \widetilde{t}) \\ \vartheta^{2}=\exp \mu_{2} d \widetilde{x}^{2} \\ \vartheta^{3}=\exp \mu_{3} d \widetilde{x}^{3}\end{array}\right)$, as a shorthand for the collection of the $\vartheta^{b}=e^{b}{ }_{\mu} d \widetilde{x}^{\mu}$, whose values at every point form the dual basis, such that $\left.e_{a}\right\rfloor \vartheta^{b}=\delta_{a}^{b}$, where $\rfloor$ denotes the interior product, namely, this is a $C^{\infty}$-bilinear map $\rfloor: \Omega^{1} \rightarrow \Omega^{0}$ with $\Omega^{p}$ denoting the $C^{\infty}$-modulo of differential p-forms on $V_{4}$. In components $e_{a}{ }^{\mu} e^{b}{ }_{\mu}=\delta_{a}^{b}$. One can also consider general transformations of a local Lorentz group, taking any frame field, $e$, into any other set, $e^{\prime}$, of four linearly independent fields: $e_{a}^{\prime}=\Lambda_{a}^{b} e_{b}$. Here and throughout the notation, $\left\{e_{a}, \vartheta^{b}\right\}$, will be used for general linear frames.

The norm $d \widetilde{s}$ then reads

$$
d \widetilde{s}:=e_{a} \vartheta^{a}=e_{\mu} \otimes \vartheta^{\mu} \in V_{4},
$$

and the holonomic metric on the space $V_{4}$ can be recast into the form

$$
g=o_{a b} \vartheta^{a} \otimes \vartheta^{a}=g_{\mu \nu} \vartheta^{\mu} \otimes \vartheta^{\nu},
$$

with the components $g_{\mu \nu}=g\left(e_{\mu}, e_{\nu}\right)(12)$ in dual holonomic basis $\left\{\vartheta^{\mu} \equiv d \widetilde{x}^{\mu}\right\}$, and $o_{a b}$ denotes $\operatorname{diag}(+-$ $--)$ metric. In the case at hand, the metric function $\omega$ is the angular velocity of the local ZAMO with respect to an observer at rest at infinity. Thereby the redshift factor $\alpha \equiv \exp \nu$ is the time dilation factor between the proper time of the local ZAMO and coordinate time $t$ along a radial coordinate line, i.e. the redshift factor for the time-slicing of a spacetime.

Given a height-function $\widetilde{t}$, the time-like unit normal to $\Sigma_{t}$ will be denoted by $n^{\mu}$ and the $3+1$ decomposition of the evolution vector field by $\widetilde{t}^{\mu}=N n^{\mu}+\beta^{\mu}$, where $N$ is the lapse function and $\beta^{\mu}$ is the shift 
vector. The induced metric on the space-like 3-slice $\Sigma_{t}$ is expressed as $\gamma_{\mu \nu}=g_{\mu \nu}+n_{\mu} n_{\nu}$, with $D_{\mu}$ the associated Levi-Civita connection and volume element ${ }^{3} \epsilon=\sqrt{\gamma} d \widetilde{x}^{1} \wedge d \widetilde{x}^{2} \wedge d \widetilde{x}^{3}$, so that ${ }^{3} \epsilon_{\mu \nu \rho}=n^{\sigma 4} \epsilon_{\sigma \mu \nu \rho}$. The extrinsic curvature of $\left(\Sigma_{t}, \gamma_{\mu \nu}\right)$ in $V_{4}$ reads $K_{\mu \nu}:=-(1 / 2) \mathcal{L}_{n} \gamma_{\mu \nu}=-\gamma_{\mu}{ }^{\rho} \nabla_{\rho} n_{\nu}$, where $\mathcal{L}$ denotes Lie derivative. Accordingly, all the geometrical objects are split into corresponding components with respect to this time-slice of spacetime. In particular, the splitting of manifold $V_{4}$ into a foliation of three-surfaces will induce a corresponding splitting of the affine connection, curvature and, thus, of the energy-momentum tensor.

The $3+1$ decomposition of the (matter) stress-energy tensor, measured by an adapted Eulerian observer of four-velocity $n^{\mu}$ in rest with respect to the foliation $\left\{\Sigma_{t}\right\}$, is $\widetilde{T}_{\mu \nu}=\widetilde{E} n_{\mu} n_{\nu}+\widetilde{p}_{(\mu} n_{\nu)}+\widetilde{S}_{\mu \nu}$, where the matter energy and momentum densities are given by $\widetilde{E}:=\widetilde{T}_{\mu \nu} n^{\mu} n^{\nu}$ and $\widetilde{p}_{\mu}:=-\widetilde{T}_{\nu \rho} n^{\nu} \gamma_{\mu}^{\rho}$, respectively, whereas the matter stress tensor is $\widetilde{S}_{\mu \nu}:=\widetilde{T}_{\rho \sigma} \gamma^{\rho}{ }_{\mu} \gamma^{\sigma}{ }_{\nu}$. Latin indices running in $\{1,2,3\}$ will be employed in expressions only involving objects intrinsic to space-like $\Sigma_{t}$ slices. That is, $\widetilde{T}^{\alpha \beta}=$ $\widetilde{E} n^{\alpha} n^{\beta}+n^{\alpha} \widetilde{J}^{\beta}+\widetilde{J}^{\alpha} n^{\beta}+\widetilde{S}^{\alpha \beta}$. Here $n^{\alpha}$ is the unit orthogonal vector to the hypersurface $\Sigma_{t}$, whereas the spacetime metric $g$ induces a first fundamental form with the spatial metric $\gamma_{\alpha \beta}$ on each $\Sigma_{t}$ as $\gamma_{\alpha \beta}=g_{\alpha \beta}+n_{\alpha} n_{\beta}$. The form (12) includes one gauge freedom for the coordinate choice. For the spherical type coordinates $\widetilde{x}^{2}=\widetilde{\theta}$ and $\widetilde{x}^{3}=\widetilde{r}$, for example, so-called quasi-isotropic gauge corresponds to $\gamma_{r \theta}=0$ and $\gamma_{\theta \theta}=\widetilde{r}^{2} \gamma_{r r}$. Then, one may define the second fundamental form which associates with each vector tangent to $\Sigma_{t}$, and the extrinsic curvature of the hypersurface $\Sigma_{t}$ as minus the second fundamental form.

Aftermath, one can define the usual Lorentz factor $W=-n_{\mu} \widetilde{u}^{\nu}=\alpha \widetilde{u}^{t}$ for a fluid which is the source of the gravitational field, with conventional stress-energy tensor

$$
\widetilde{T}^{\mu \nu}=(\widetilde{\rho}+\widetilde{P}) \widetilde{u}^{\mu} \widetilde{u}^{\nu}+\widetilde{P} g^{\mu \nu},
$$

where $\widetilde{\rho}$ is the total energy density and $\widetilde{P}$ is the pressure. Hence $\widetilde{E}=W^{2}(\widetilde{\rho}+\widetilde{P})-\widetilde{P}$ and $\widetilde{J}^{i}=$ $(\widetilde{E}+\widetilde{P}) \widetilde{v}^{i}$, where the fluid three-velocity $\widetilde{v}^{i}(i=1,2,3)$ implies $\widetilde{u}^{i}=W\left(\widetilde{v}^{i}-\beta^{i} / \alpha\right)$. Thereby the resulting stress tensor can be written as $\widetilde{S}_{i j}=(\widetilde{E}+\widetilde{P}) \widetilde{v}_{i} \widetilde{v}_{j}+\widetilde{P} \gamma_{i j}$. The four-velocity for rotating fluid reads $\widetilde{u}=\widetilde{u}^{i}(\partial / \partial \widetilde{t})+\Omega \partial / \partial \widetilde{\phi}$, where $\Omega=\widetilde{u}^{\phi} / \widetilde{u}^{t}$ is the fluid angular velocity as seen by an inertial observer at rest at infinity. Consequently, the components of the energy - momentum tensor of matter with total density $\rho$ and pressure $P$ are given in the non-rotating anholonomic orthonormal frame as $\widetilde{T}^{(a b)}=$ $e_{\mu}^{a} e_{\nu}^{b} \widetilde{T}^{\mu \nu}, \quad \widetilde{T}^{(00)}=W^{2}\left(\widetilde{\rho}+\widetilde{P} V^{2}\right), \quad \widetilde{T}^{(11)}=W^{2}\left(\widetilde{\rho}+\widetilde{P} V^{2}\right), \quad \widetilde{T}^{(01)}=W^{2}(\widetilde{\rho}+\widetilde{P}) V$ and $\widetilde{T}^{(22)}=\widetilde{T}^{(33)}=\widetilde{P}$, with its trace $\widetilde{T}=-\widetilde{\rho}+3 \widetilde{P}$, where $V$ is the velocity (in units of c) with respect to the Bardeen observer $V=\varrho B(\Omega-\omega) / \alpha^{2}$, so $W=1 / \sqrt{1-V^{2}}$.

\section{Field Equations}

In the case of stationary and axisymmetric space $V_{4}$, the equations of $1 \mathrm{D}$ gravitation $x_{0}$, the framedragging potential $x_{1}$, and the ID field $\bar{x}$, generated by the stress-energy tensor (15) of isolated spinning fluid, can be obtained from (68) in the Feynmann gauge:

$$
\Delta x_{0}(x)=-\widetilde{J}_{\left(x_{0}\right)}(\widetilde{x}), \quad \Delta x_{1}(x)=-\widetilde{J}_{\left(x_{1}\right)}(\widetilde{x}), \quad\left(\Delta-\lambda_{\bar{x}}^{-2}\right) \bar{x}(x)=-\widetilde{J}_{(\bar{x})}(\widetilde{x}) .
$$

Here the current $\widetilde{J}_{(\chi)}\left(\chi:=x_{0}, x_{1}, \bar{x}\right)$ is referred to as

$$
\widetilde{J}_{(\chi)}:=(\kappa / 2) \sqrt{-g} e_{a}^{\mu} e_{b}^{\nu} \frac{\partial g_{\mu \nu}}{\partial \chi} \widetilde{T}^{(a b)},
$$

provided, the Compton length of the ID-field $\bar{x}$ is $\lambda_{\bar{x}}=\left(\hbar / m_{\bar{a}} c\right) \simeq 0.4 \mathrm{fm}$.

The system of non-linear differential field equations (16) explicitly reads

$$
\begin{aligned}
& \Delta x_{0}=-\frac{\kappa}{2} \sqrt{-g}\left[\exp (-2 \nu) W^{2}\left(\widetilde{\rho}+\widetilde{P} V^{2}\right) \times \frac{\partial g_{00}}{\partial x_{0}}+\exp \left(-2 \mu_{3}\right) \widetilde{P} \frac{\partial g_{33}}{\partial x_{0}}\right], \\
& \Delta x_{1}=-\frac{\kappa}{2} \sqrt{-g} \exp (-\nu) \frac{\partial g_{01}}{\partial x_{1}}\left[\exp (-\nu) \omega \times W^{2}\left(\widetilde{\rho}+\widetilde{P} V^{2}\right)+\exp (-\psi) W^{2}(\widetilde{\rho}+\widetilde{P}) V\right], \\
& \left(\Delta-\lambda_{\bar{x}}^{-2}\right) \bar{x}=-\frac{\kappa}{2} \sqrt{-g}\left[\exp (-2 \nu) W^{2}\left(\widetilde{\rho}+\widetilde{P} V^{2}\right) \frac{\partial g_{00}}{\partial \bar{x}}+\exp \left(-2 \mu_{3}\right) \widetilde{P} \frac{\partial g_{33}}{\partial \bar{x}}\right] .
\end{aligned}
$$


These equations can be solved together with a diffeomorphism $\widetilde{x}^{\mu}(x): M_{4} \rightarrow V_{4}(11)$. Adopting spherical polar coordinates $(r, \theta, \phi)$ in flat space $M_{4}$, the (11) gives

$$
\frac{\partial \widetilde{r}}{\partial r}=\psi_{3}^{\widetilde{3}}=D_{3}^{\widetilde{3}}(1+\varpi), \quad \frac{\partial \widetilde{r}}{\partial \theta}=\psi_{2}^{\widetilde{3}}=0, \quad \frac{\partial \widetilde{\theta}}{\partial r}=\psi_{3}^{\widetilde{2}}=0, \quad \frac{\partial \widetilde{\theta}}{\partial \theta}=\psi_{2}^{\widetilde{2}}=D_{2}^{\widetilde{2}}(1+\varpi)
$$

Hence $d \widetilde{r}=\psi_{3}^{\widetilde{3}}(\widetilde{r}, \widetilde{\theta}) d r$ and $d \widetilde{\theta}=\psi_{2}^{2}(\widetilde{r}, \widetilde{\theta}) d \theta$, which means that $r=r(\widetilde{r}, \widetilde{\theta})$ and $\theta=\theta(\widetilde{r}, \widetilde{\theta})$. Therefore, the stationary axisymmetric field components $\chi(r, \theta)$ are the solutions of the system of non-linear differential axisymmetric Poisson field equations (18). Recall that the infinite series expansion of the factor $1 /\left|\mathbf{x}-\mathbf{x}^{\prime}\right|$ in Green function of Poisson equation in spherical polar coordinates $(r, \theta, \phi)$, in general, is

$$
\frac{1}{\left|\mathbf{x}-\mathbf{x}^{\prime}\right|}=\Sigma_{l=0}^{\infty} \frac{r_{<}^{l}}{r_{>}^{l+1}} P_{l}(\cos \theta) P_{l}\left(\cos \theta^{\prime}\right)+(\phi-\text { dependent terms }),
$$

where $P_{l}$ denotes a Legendre polynomial and $r_{<}\left(r_{>}\right)$is the lesser (greater) of $r$ and $r^{\prime}$. By azimuthal symmetry, the $\phi$-dependant terms do not contribute to the $(20)$.

\section{A Global Vacuum Solution: Horizons}

The existence of a global vacuum solution, $\chi\left(\equiv x_{0}, x_{1}\right)$, outside of the matter, amounts to solving the field equations (16) in a nearly Newtonian weak source limit: $\widetilde{T} \rightarrow 0, \bar{x}=0$. In Lorentz gauge, it is given by means of fundamental solution of Poisson's equation as a retarded integral of the form familiar from linearized field equation theory:

$$
\chi(\mathbf{x})=\frac{1}{4 \pi} \int \frac{\widetilde{J}_{(\chi)}^{e x t}\left(\left|\mathbf{x}-\mathbf{x}^{\prime}\right|\right) d^{3} x^{\prime}}{\left|\mathbf{x}-\mathbf{x}^{\prime}\right|}
$$

where the current $\widetilde{J}_{(\chi)}^{\text {ext }}\left(\left|\mathbf{x}-\mathbf{x}^{\prime}\right|\right)$ denotes

$$
\widetilde{J}_{(\chi)}^{e x t}\left(\left|\mathbf{x}-\mathbf{x}^{\prime}\right|\right)=4 \pi Q_{(\chi)}\left(\left|\mathbf{x}-\mathbf{x}^{\prime}\right|\right) \delta\left(\left|\mathbf{x}-\mathbf{x}^{\prime}\right|\right) .
$$

The charge $Q_{(\chi)}\left(\left|\mathbf{x}-\mathbf{x}^{\prime}\right|\right)$ is calculated in the spacetime region far outside the system, where we assume that the spacetime is almost Minkowski $g \simeq \eta$. The external metric $g^{\text {ext }}=g^{\text {ext }}(\chi)$ is then written as

$$
\begin{aligned}
& g_{00}^{e x t}=\tau_{1}^{2}\left(1-\tan ^{2} \theta_{1}\right)+\tau_{2}^{2} \cos 2 \theta_{1}+\tau_{3}^{2}\left[\cos ^{2} \theta_{1}\left(1-x_{0}\right)^{2}-\sin ^{2} \theta_{1}\left(1+x_{0}\right)^{2}\right], \\
& g_{01}^{e x t}=-2 \tau_{1} x_{1}, \quad g_{11}^{e x t}=-\left(1+\tan ^{2} \theta_{1}\right), \quad g_{22}^{e x t}=-\cos 2 \theta_{1}, \\
& g_{33}^{e x t}=-\cos ^{2} \theta_{1}\left(1+x_{0}\right)^{2}+\sin ^{2} \theta_{1}\left[\left(1-x_{0}\right)^{2} .\right.
\end{aligned}
$$

The Petrov type D vacuum solutions (21)-(23) are associated with the gravitational field of isolated massive stationary and axisymmetric rotating SPC. They are completely characterized by its mass $M_{S P C}$ and angular momentum $J_{S P C}$.

The two double principal null directions define "radially" ingoing and outgoing null congruences near the SPC which is the source of the field. The horizon is a $2 \mathrm{D}$ surface of spherical topology, where the redshift factor $\alpha(r, \theta)$ vanishes

$$
g_{00}^{e x t}(r, \theta)=\alpha^{2}(r, \theta)=\tau_{1}^{2}\left(1-\tan ^{4} \theta_{1}\right)+\tau_{2}^{2}\left(1-\tan ^{2} \theta_{1}\right)+\tau_{3}^{2}\left[\left(1-x_{0}\right)^{2}-\tan ^{2} \theta_{1}\left(1+x_{0}\right)^{2}\right]=0 .
$$

The gravitational infinite redshift suppresses any emission at the horizon. The solution of (24), for given $x_{1}$, is

$$
x_{0}^{( \pm)}=\left(\tau_{3}\left(1-x_{1}^{2}\right)\right)^{-1}\left\{\tau_{3}\left(1+x_{1}^{2}\right) \pm \sqrt{\tau_{3}^{2}\left(1+x_{1}^{2}\right)^{2}-\left(1-x_{1}^{2}\right)\left(1-\tau_{2}^{2} x_{1}^{2}-\tau_{1}^{2} x_{1}^{4}\right)}\right\},
$$

where the discriminant has to be positive.

(i) At $x_{1}<1$, the gravitational field potentials $x_{0}^{( \pm)}$(25) yield two physical horizons. Their radii coincide with $r_{g}=R_{g} / 2\left(x_{0}^{( \pm)}=1\right)$ on the axis of rotation of SPC, located at $\theta=0$ (where $\left.x_{1}=0\right)$, where $R_{g}=2 G M / c^{2}=2.95 \times 10^{5} M / M_{\odot} \mathrm{cm}$ is the Schwarzschild radius (radius of a non-rotating black hole).

(ii) At $x_{1}\left(r_{0}\right)=1$, the outer oblate horizon can be formed only at infinity $r_{0} \rightarrow \infty$, where $x_{0}\left(r_{0} \rightarrow\right.$ $\infty) \rightarrow 0$. 
(iii) For vanishing $x_{1} \rightarrow 0$, the solution (25), at $\tau_{3} \rightarrow 1\left(\tau_{2}=\tau_{1} \rightarrow 0\right)$, becomes static, spherically symmetric, yielding a single spherical event horizon $x_{0}^{( \pm)}(r) \rightarrow 1$ of previous model of non-rotating SPC ([9]-[12],[1].

The horizons are null surfaces because they are light-like. The hypersurfaces with $r_{ \pm}$are Killing horizons of the Killing vector field. A null hypersurface $\mathcal{N}$ is a Killing horizon of a Killing vector $\xi$ if, on $\mathcal{N}, \xi$ is normal to $\mathcal{N}$ [27]. Suppose we adopt affine parametrization $l \cdot D l^{\mu}=0$. Since $\xi=f l$ on $\mathcal{N}$ for some function $f$, it follows that $\xi \cdot D \xi^{\mu}=k \xi^{\mu}$ on $\mathcal{N}$, where $k=\xi \cdot \partial \ln |f|$ is called the surface gravity. Since $\xi$ is normal to $\mathcal{N}$, Frobenius theorem implies that $\left.\xi_{[\mu} D_{\nu} \xi_{\rho]}\right|_{\mathcal{N}}=0$, where '[...]' indicates total anti-symmetry in the enclosed indices. For a Killing vector field $\xi$, it implies $D_{\mu} \xi_{\nu}=D_{[\mu} \xi_{\nu]}$. Except at points for which $\xi=0$, one then has $k^{2}=\left.(1 / 2)\left(D^{\mu} \xi^{\nu}\right)\left(D_{\mu} \xi_{\nu}\right)\right|_{\mathcal{N}}$. All points at which $\xi=0$ are limit points of orbits of $\xi$ for which $\xi \neq 0$, so continuity implies that this formula is valid even when $\xi=0$.

One can then prove that $k$ is constant on orbits of $\xi: \xi \cdot \partial k^{2}=-\left(D^{\mu} \xi^{\nu}\right) R_{\nu \mu \rho \sigma} \xi^{\rho} \xi^{\sigma}=0$, because of antisymmetry $R_{\nu \mu \rho \sigma}=-R_{\nu \mu \sigma \rho}$. The surface gravity $k$ is not a property of $\mathcal{N}$ alone, it also depends on the normalization of $\xi$, because if $\mathcal{N}$ is a Killing horizon of $\xi$ with surface gravity $k$, then it is also a Killing horizon of $c \xi$ with surface gravity $c^{2} k$ for any constant $c$. There is no natural normalization of $\xi$ on $\mathcal{N}$ since $\xi^{2}=0$ there, but in an asymptotically flat spacetime there is a natural normalization at spatial infinity, e.g. for the time-translation Killing vector field $k$ we choose $k^{2} \rightarrow-1$ as $r \rightarrow \infty$. This fixes $k$, and hence $k$, up to a sign, and the $\operatorname{sign}$ of $k$ is fixed by requiring $k$ to be future-directed.

\subsection{External Fields in the Weak Source Limit}

The external fields $\chi\left(\left|\mathbf{x}-\mathbf{x}^{\prime}\right|\right)(21)$ can be obtained straightforwardly in the weak source limit $\widetilde{T} \rightarrow 0$, where the spacetime is almost Minkowski $g \simeq \eta$. It is sufficient then to consider an isolated system only in the asymptotic linearized regime. Without loss of accuracy, one can therefore keep only leading linear order-terms of fields, ignoring a second and higher order effects. Linearized field equations guarantee conservation of 4-momentum and angular momentum of any body bounded by vacuum. So, we expand the solutions $\chi\left(\left|\mathbf{x}-\mathbf{x}^{\prime}\right|\right)$ in powers of $x^{\prime} / r=x^{\prime} /|\mathbf{x}|$. It is convenient to perform calculation in the system's rest frame, where $P^{j}=\int T^{j 0} d^{3} x=0$, with origin of coordinates at the system's center of mass $\int x^{j} T^{00} d^{3} x=0$. Hence, in suitable asymptotically Minkowski coordinates, where the stationary Killing vector is given by $m=\partial_{t}$, the resulting redshift factor $\alpha(M, r)$ and the frame-dragging potential $\omega(J, r)$ can be defined by means of the constants $M$ and $J$, respectively, as the total mass and intrinsic angular momentum of the source.

These quantities are well substantiated by Komar integral [28]. Recall that to every Killing vector field $\xi$ in the volume $V$ of spacetime on a spacelike hypersurface $\Sigma$, with boundary $\partial V$, one can associate the Komar integral

$$
Q_{\xi}(V)=(q / 16 \pi G) \oint d S_{\mu \nu} D^{\mu} \xi^{\nu},
$$

with some constant $q$. Since Killing fields satisfy the identity $D_{\nu} D_{\mu} \xi^{\nu}=R_{\mu \nu} \xi^{\nu}$, where $R_{\mu \nu}$ is the curvature tensor of $V_{4}$, then $Q_{\xi}(V)=\int_{V} d S_{\mu} J_{(\xi)}^{\mu}$, and the current $J_{(\xi)}^{\mu}$ is defined as follows: $J_{(\xi)}^{\mu}:=q R_{\nu}^{\mu} \xi^{\nu}$. The current $J_{(\xi)}^{\mu}$ is conserved $D_{\mu} J_{(\xi)}^{\mu}=0$, and the charge $Q_{\xi}(V)$ is time-independent, provided $J_{(\xi)}^{\mu}$ vanishes on the boundary $\partial V$. If $\xi=k, q=-2$ is fixed by comparison with the formula derived for total mass (energy) in asymptotic expansion, the $M$ integral can be recast into a coordinate-independent form

$$
M=-(1 / 8 \pi G) \oint_{\infty} d S_{\alpha \beta} D^{\alpha} k^{\beta} .
$$

If $m=\partial_{t}$ is the Killing vector of axisymmetry, one obtains for $q=1$ a coordinate-independent angular momentum integral

$$
J=(1 / 16 \pi G) \oint_{\infty} d S_{\alpha \beta} D^{\alpha} m^{\beta} .
$$

For a weak source, $g \simeq \eta$, the total mass (27) and angular momentum (28) of an asymptotically flat spacetime is found by taking $\partial V$ to be a 2 -sphere at spatial infinity.

If one chooses $V$ to be on $t$ constant hypersurface, then in Cartesian coordinates $x^{i}(i=1,2,3)$, one has $d S_{\mu} m^{\mu}=0$ and $m=x^{1} \partial_{2}-x^{2} \partial_{1}$. For a weak source, therefore $J(V) \simeq \varepsilon_{3 i j} \int_{V} x^{i} T^{j 0}$. Using Killing equations $D_{(\alpha} k_{\beta)}=0=D_{(\alpha} m_{\beta)}$, which lead to $D_{\alpha} D^{\alpha} k^{\beta}=R_{\rho}^{\beta} k^{\rho}$ and $D_{\alpha} D^{\alpha} m^{\beta}=R^{\beta}{ }_{\rho} m^{\rho}$, the $(27)$ and (28) can be converted by means of Stoke's theorem as

$$
M=-(1 / 4 \pi G) \int_{\Sigma} R^{\alpha}{ }_{\beta} k^{\beta} d \Sigma_{\alpha}, \quad J=(1 / 8 \pi G) \int_{\Sigma} R_{\beta}^{\alpha} m^{\beta} d \Sigma_{\alpha} .
$$


The leading linear order-terms in asymptotic expansion of the redshift factor and the frame-dragging potential are given by

$$
\alpha\left(R_{g}, r\right) \simeq 1-R_{g} / 2 r+O\left(1 / r^{3}\right), \quad \omega(J, r)=2 G J / c^{3} r^{3}+O\left(1 / r^{4}\right) .
$$

Equating these values respectively to the functions $\left[g_{00}^{e x t}(M, r)\right]^{1 / 2}$ and $\left[-(1 / 2) g_{01}^{e x t}(J, r)\right]$, where the components of the external metric (23), which are accurate to linearized order, become $g_{00}^{\text {ext }} \simeq 1-2 \tau_{3}^{2} x_{0}$, and $g_{01}^{e x t}=-2 \tau_{1} x_{1}$, we finally obtain

$$
x_{0}(r)=R_{g} / 2 \tau_{3}^{2} r, \quad x_{1}(r)=2 G J_{S P C} / \tau_{1} c^{3} r^{3} .
$$

The potential of dragging of inertial frames $\omega$ drops very rapidly with increasing radius (31). The maximal frame-dragging effects therefore can only be observed in the immediate vicinity of the event horizon:

$$
x_{1(H)}=\omega_{H} / \tau_{1} \simeq 2 G J_{S P C} / \tau_{1} c^{3} R_{g}^{3}=J_{S P C} c^{3} / 4 \tau_{1} G^{2} M^{2} .
$$

In physical units the angular momentum $J_{S P C}=a M_{S P C}$ becomes $J_{S P C}=a_{*} M_{S P C} \frac{G M_{S P C}}{c^{2}}$, such that $x_{1(H)}=\left(2 c a_{*} / \tau_{1} G M_{S P C}\right)$, where $a_{*}$ is the dimensionless angular momentum $\left|a_{*}\right| \leq 1$. The special case $a=M_{S P C}$ is the extreme solution. From this we get the maximal specific angular momentum $J_{S P C}^{\max }=\left(G M_{S P C} / c\right)$, and that $x_{1(H)}=2 a_{*} / \tau_{1} J_{S P C}^{\max }<1$.

The Petrov type D vacuum solutions for stationary axisymmetric rotating SPC, therefore, satisfy the Robinson's theorem for Kerr solutions in vacuum [29]: the solutions, (i)-are asymptotically flat, (ii)contain a smooth convex horizon, (iii)- are nonsingular outside the horizon, and are uniquely specified by two parameters: the mass $M_{S P C}$ and angular momentum $J_{S P C}$. The angular velocity of a SPC is the sum of two terms: the classical one given by the intrinsic angular velocity $\Omega$ and the frame dragging $\omega$ from the rotation of absolute space. Near the horizon of SPC, for example, where the redshift tends to zero $(\alpha \rightarrow 0)$, the angular velocity of matter $\Omega$ is completely dominated by the frame-dragging effect.

Whatever the intrinsic angular momentum of the incoming matter is, this matter is forced to rotate with the local angular velocity $\omega$, which is the maximal angular velocity at event horizon. When matter falls, say into a nonrotating black hole, it is forced to zero rotation near the horizon despite its angular momentum. The event horizon is a Killing horizon for the Killing field $\xi=k+\Omega_{H} m$, with $\xi^{2}=0$, where $\Omega_{H}$ denotes the angular velocity of the horizon as it is rigidly rotating.

Let $m=\partial_{t}$ be the axial Killing field, and $\widetilde{u}=\widetilde{u}^{t}\left(1, \Omega_{H}, 0,0\right)$ be the four-velocity of SPC. We may choose $\tau_{2}=0, \tau_{1}=\tau_{3}=1 / \sqrt{2}$. Since $x_{1(H)}<1$, analogous to the Kerr black hole, there are the outer horizon or event horizon by its radius $r_{+}$, and the inner horizon or Cauchy horizon by its radius $r_{-}$. The radii $r_{+}$and $r_{-}$coincide with $r_{g}\left(x_{0}^{( \pm)}=1\right)$ on the axis of rotation of SPC, located at $\theta=0\left(J_{S P C}=0\right)$. The oblate event horizon is the surface of the oblate spheroid with the semimajor $r_{+}$and semiminor $r_{g}$ axes, respectively. Dependent of the value of the potential $x_{1(H)}$, the Cauchy horizon either is the surface of the prolate spheroid with the semimajor $r_{g}$ and semiminor $r_{-}$axes, or oblate spheroid with the semimajor $r_{-}$and semiminor $r_{g}$ axes, respectively. The radii of the oblate event horizon and the Cauchy horizon are given by $r_{\mp}=r_{g} / \tau_{3}^{2} x_{0}^{( \pm)}$, provided with (25). These radii, as the function of $x_{1(H)}$, are plotted in Fig. 1.

Since $\alpha\left(r_{+}\right)=0$, any observer, but the ZAMO, rotates at the outer horizon with the angular velocity $\Omega_{H}$. The angular velocity of ZAMO is $\Omega=\omega$, which will have vanishing specific angular momentum:

$$
\widetilde{u}^{\phi}=g_{\phi \alpha} \widetilde{u}^{\alpha}=g_{\phi \phi} \widetilde{u}^{\phi}+g_{t \phi} \widetilde{u}^{t}=\widetilde{u}^{t}\left(\omega g_{\phi \phi}+g_{t \phi}\right)=\widetilde{u}^{t} g_{\phi \phi}\left(\omega+g_{t \phi} / g_{\phi \phi}\right)=0 .
$$

It is therefore convenient to express physical observables with respect to ZAMO.

In the slow-rotation limit, the angular momentum of SPC, as rigidly rotating body, can be given in the form

$$
J=\int_{M_{H}}(\widetilde{\rho}+\widetilde{P})\left(\widetilde{u}^{t}\right)^{2}\left(g_{t \phi}+\Omega g_{\phi \phi}\right) \sqrt{-g} d^{3} \widetilde{x} \simeq \int_{M_{H}}(\widetilde{\rho}+\widetilde{P})\left(\widetilde{u}^{t}\right)^{2} g_{\phi \phi}\left(\Omega_{H}-x_{1(H)} / \sqrt{2}\right) \sqrt{-g} d^{3} \widetilde{x},
$$

which in leading order in the expansion for $\Omega_{H}$ reads

$$
J \simeq \frac{8 \pi}{3} \int_{0}^{R_{S P C}} r^{4} \frac{(\rho+P)}{\left(1-r_{g} / r\right) \sqrt{1-2 M(r) / r}}\left(\Omega_{H}-2 a_{*} / 4 J_{S P C}^{\max }\right) d r
$$

where $R_{S P C}$ is the radius of SPC. 


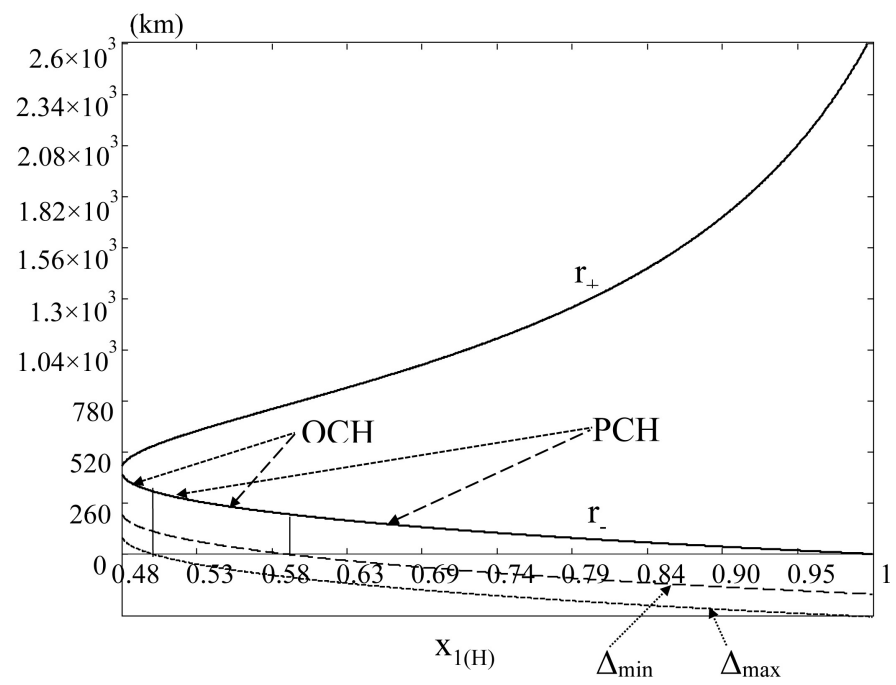

Figure 1. The radii $r_{ \pm}$of outer and inner horizons v.s. potential $x_{1(H)}$, for $\mathrm{M} 82 \mathrm{X}-2$, with the mass $M \simeq$ $138.5-226 M_{\odot}[1]$, i.e. $r_{g}=R_{g} / 2=r_{g}^{\max }-r_{g}^{\min } \simeq 204.3-333.4 \mathrm{~km}$.

Abbreviated notations: $\Delta_{\min }:=r_{-}-r_{g}^{\min }, \Delta_{\max }:=r_{-}-r_{g}^{\max }$, OCH $:=$ Oblate Cauchy Horizon, PCH $:=$ Prolate Cauchy Horizon.

\section{Microscopic Model of Stationary and Axisymmetric Rotating SPC}

The entire set of the equations, describing the stationary and axisymmetric rotating SPC-configuration, includes the field equations (18), the hydrostatic equilibrium equation and the state equation of the spherical-symmetric distribution of barionic matter in many-phase stratified states. A layering is a consequence of the onset of different regimes in equation of state. A rigorous numerical solution of these equations will be a separate topic of investigation elsewhere. But note that the key physical properties of SPC depend very little on the details of concrete SPC-model, as they are a direct consequence of the fundamental features of underlying gravitation theory. The lattter explores a spontaneous breaking of gravitation gauge symmetry and a rearrangement of vacuum state. We therefore expect that the key properties of non-rotating SPC, outlined in subsection 1.1, even though without being carefully treated, retain for a rotating $\mathrm{SPC}$ too.

Below we extend preceding developments of previous model of non-rotating SPC, without going into the subtleties, as applied to the initially rigid-body rotating SPC of angular velocity $\Omega_{S P C}$. A microscopic model makes room for growth and merging behavior of black holes. The black hole seeds might grow driven by the accretion of outside matter when they were getting most of their masses.

An infalling matter with time forms a thin co-spinning proto-matter disk which has reached out the edge of the outer oblate event horizon. Since a metric singularity inevitably disappears, the ZeV-neutrinos, as in previous non-rotating case produced via simple or modified URCA processes in deep layers of SPC and proto-matter disk, may escape from event horizon to outside world. The neutrinos are collimated in very small opening angle. In this framework we introduce a notion of pre-radiation time (PRT) of black hole which is referred to as a lapse of time $T_{B H}$ from the birth of black hole till neutrino radiation - provision for the first half of the lifetime of black hole: $T_{B H}=\frac{M_{d}}{\dot{M}}$. Here $M_{d}$ is the mass of proto-matter disk, $\dot{M}$ is an appropriately averaged mass accretion rate. The typical PRT versus bolometric luminosity of suppermassive black holes is given by

$$
T_{B H} \simeq 0.32 \frac{R_{d}}{r_{O V}}\left(\frac{M_{B H}}{M_{\odot}}\right)^{2} \frac{10^{39} W}{L_{b o l}} \mathrm{yr},
$$

where $R_{d}$ is the radius of the proto-matter core, $r_{O V}=13.68 \mathrm{~km}$. Some evidence for a simplified physical picture, for example, at $x_{1}<1$ in the weak source limit (iii), is highlighted in Fig. 2 to guide the eye, without loss of generality. 

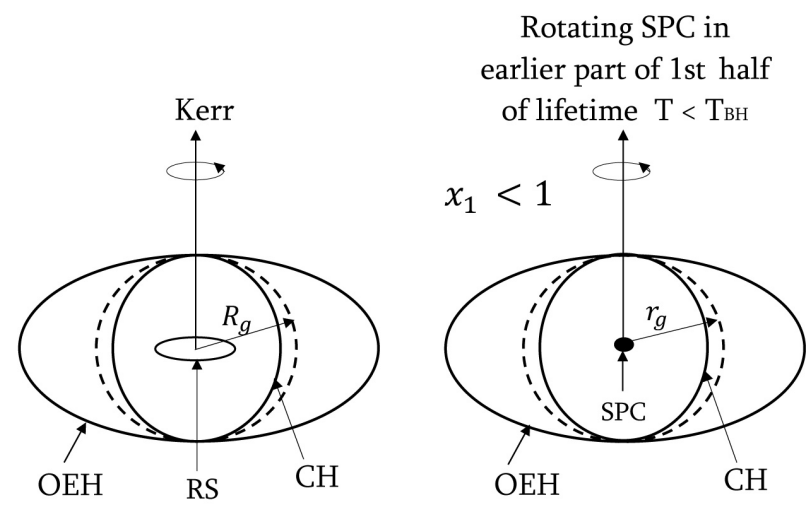

Figure 2. Left panel: Kerr model of spinning black hole. The meaningless ring singularity occurs at the center inside the black hole. Right panel: Microscopic model of rotating SPC in earlier part of first half of its lifetime $T<T_{B H}$. The picture is not to scale.

Abbreviated notations: OEH :=Oblate Event Horizon, SPC :=Superdense Proto-matter Core, RS :=Ring Singularity, $\mathrm{PCH}:=$ Prolate Cauchy Horizon.

In the first half of its lifetime, the external physics outside of outer oblate event horizon of accretion onto the SPC hole is very closely analogous to the processes in Kerr's model. But a crucial difference between Kerr and microscopic models is the interior solutions. However, the interior solution of MTBH is physically meaningful, because it has smeared out a central ring singularity of the Kerr black hole replacing it by the equilibrium SPC inside event horizon, where the static observers exist.

This ultimately circumvent the principle problem of an observer's inability to access the degrees of freedom that are hidden beyond the horizon. The physical entropy of SPC is a measure of the large number of the real thermodynamical microstates, which are compatible with the ergodicity.

Before attempting to build a physical model of the periodic ULX M82X-2, the other features of SIMBH configuration also need to be accounted. The event horizon is impenetrable barrier for crossing from inside the Kerr black hole. In the framework of microscopic model, this barrier disappears because the metric (9) clearly shows that: singularity at intersection of proto-matter disk with either Cauchy horizon or outer event horizon vanishes wherever the ID-field is not zero $(\bar{x} \neq 0)$, and hence the crossing of these horizons either from inside or outside of black hole at such conditions is allowed. Certainly, approaching to horizon the increase of particle concentration of infalling gas, $n$, is brought to halt when it asymptotically reaches to the threshold value, $n^{-1 / 3}=\lambda_{\bar{x}} \simeq 0.4 \mathrm{fm}$, and the ID-field $\bar{x}$ switches on and, thus, infalling gas becomes proto-matter. Then, a metric singularity vanishes as in the former case, and infalling gas passes freely through the horizon.

The Fig. 3 emphasizes an apparent distinction between Kerr model and rotating SPC in second half of its lifetime.

\section{Model Building of the Periodic Source M82X-2}

To see where all this is leading to, let us briefly return next to the real issue of the periodic ULX M82X-2. As pointed out by [1]: (i) the geodetic effect, as in the case of a gyroscope, leads an accretion stream to a tilting of its spin axis in the plain of the orbit. Hence a proto-matter disk will be tilted from the plane of accretion on a definite angle $\delta$ towards the equator.

The UHE-neutrinos, produced in the deep interior layers of superdense proto-matter medium as in case of previous non-spinning model. the additional thermal defuse blackbody radiation is released from the outer surface layers of ordinary matter of spinning SPC and co-spinning proto-matter thin disk. All of the rotational kinetic energy is dissipated as thermal blackbody radiation. This is due to the fact that these layers are optically thick and, eventually, in earlier half of the lifetime of spinning black hole, at times $<T_{B H}$, the strict thermodynamic equilibrium prevails for this radiation because there would be no net flux to outside of event horizon in any direction. That is, the emission from the isothermal, optically 
Rotating SPC in

2nd half of lifetime

$\mathrm{T} \geq \mathrm{T}_{\mathrm{BH}}$

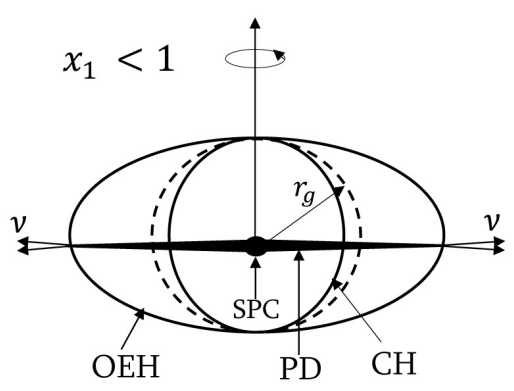

Figure 3. Microscopic model of rotating SPC in second half of its lifetime. An infalling matter already formed a thin co-spinning proto-matter disk which has reached out the edge of the outer oblate event horizon. A singularity inevitably disappears and the neutrinos escape to outside world through the vista.

Abbreviated notations: OEH $:=$ Oblate Event Horizon, SPC $:=$ Superdense Proto-matter Core, PCH $:=$ Prolate Cauchy Horizon, PD :=Proto-matter Disk.

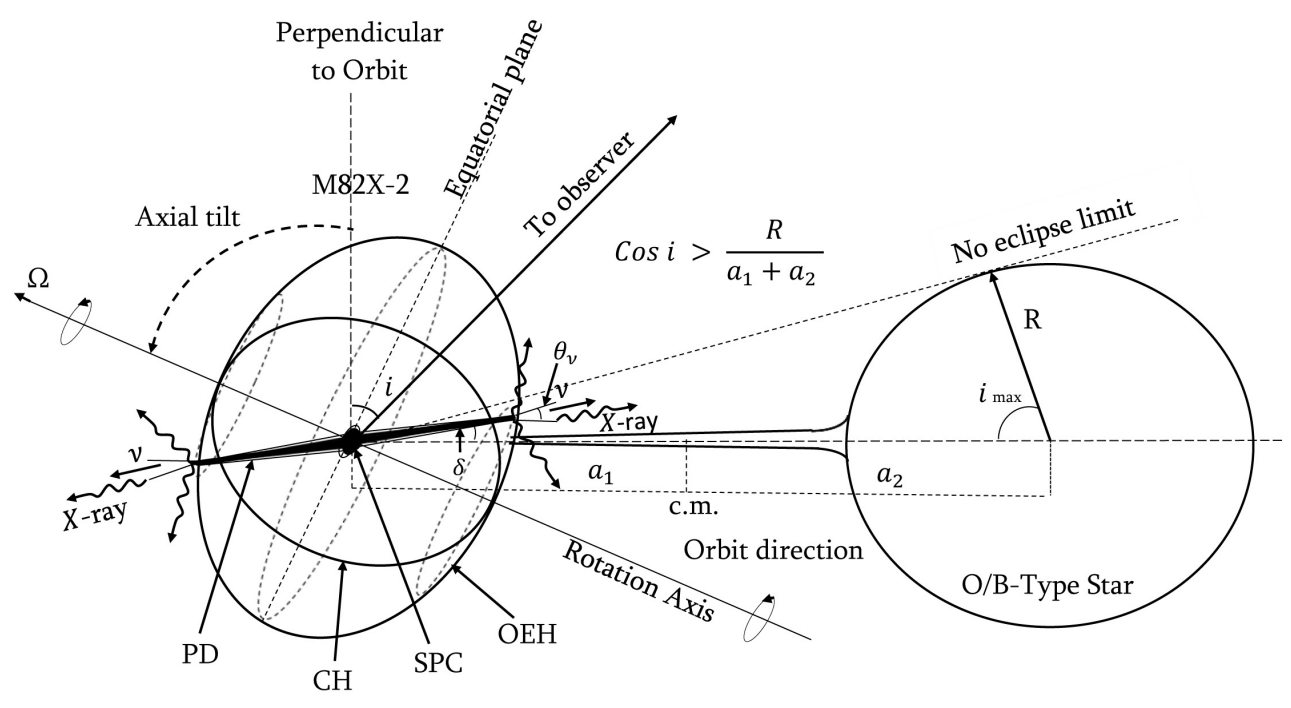

Figure 4. A schematic SIMBH model of M82X-2 constituting mass-exchange binary with the O/B-type donor star. The angle $i$ is the binary inclination with respect to the plane of the sky. No eclipse condition holds. In final stage of growth, PD has reached out the edge of the OEH. The thermal defuse blackbody X-rays beams may escape from SIMBH through a thin belt area $S=2 \pi R(\theta) d$ to outside world that sweep past Earth like lighthouse beacons. Parameters of a binary system is viewed in the orbital plane. The picture is not to scale.

Abbreviated notations: OEH :=Oblate Event Horizon, SPC :=Superdense Proto-matter Core, PCH := Prolate Cauchy Horizon, PD :=Proto-matter Disk. 
thick outer layers at surface is blackbody, which is the most efficient radiation mechanism. This radiation is free of trapping.

With this guidelines to follow, we may proceed to Fig. 4, which schematically plots the SIMBH model of the periodic ULX M82X-2, constituting mass-exchange binary with the O/B-type donor star. The OEH-surface of the spheroid has the polar equation $R=R(\vartheta)$, where

$$
\frac{r_{g}^{2}}{R^{2}}=\cos ^{2} \vartheta+\left(1-e^{2}\right) \sin ^{2} \vartheta
$$

provided, $\vartheta$ is the reduced or parametric latitude $(-\pi / 2<\vartheta<\pi / 2)$, $e$ denotes the eccentricity $e:=$ $e\left(x_{1(H)}\right)=\sqrt{1-r_{g}^{2} / r_{+}^{2}\left(x_{1(H)}\right)}$, while $r_{+}\left(x_{1(H)}\right)$ and $r_{g}:=R(0)$ are the semimajor and semiminor axes, respectively, of the rotated ellipse of $\mathrm{OEH}$.

The OEH introduces the corrections to the characteristic phase profile of previous model of X-ray radiation from M82X-2, derived in approximation of spherical EH [1]. Certainly, let (z,x,y) be the spacefixed Cartesian coordinate system, with zx as a plane-of sight, and the axis $s$ of the M82X-2-fixed frame as the rotation axis. The angles $\theta$ and $\phi$ are spherical polar coordinates. The observed pulses are produced because of periodic variations with time of the projection on the plane-of sight, $d_{z x}(t)$, of the vector $\boldsymbol{d}(t)$ collinear to $\boldsymbol{n}(t)\left(\boldsymbol{d}(t)=d \frac{\boldsymbol{n}(t)}{|n(t)|}\right)$, where $\boldsymbol{n}(t)$ is the normal to the plane of the proto-matter disk at the moment $t$. The $\boldsymbol{n}(0)$ lies in the plane of zs. The pulsations will be observed if and only if the projection of the belt area $S_{z x}=C_{1}(e, \alpha) d_{z x}(d, \theta, \phi, \alpha, t)$ on the plane of sight zx is not zero, where $C_{1}(e, \alpha)$ is the circumference of the ellipse of intersection of the proto-matter disk plane with the OEH, $\alpha$ is the angle between the equatorial plane and the proto-matter disk plane. The semi-major and semi-minor axes of this ellipse are $a_{1}=r_{+}:=R(\pi / 2), \quad b_{1}(\alpha):=R(\pi / 2+\alpha)$, respectively, such that

$$
e_{1}(\alpha)=\sqrt{1-b_{1}^{2}(\alpha) / r_{+}^{2}}=\frac{e}{\sqrt{1+\left(1-e^{2}\right) \cot ^{2} \alpha}} .
$$

In the case of $\mathrm{OEH}$, the pulsed luminosity reads

$$
\widetilde{L}\left(M, d, T_{s}, e, \theta, \phi, \alpha, t\right)=S_{z x} \sigma T_{s}^{4}=L_{0}\left(M, d, T_{s}\right) \Phi^{\prime}(e, \theta, \phi, \alpha, t),
$$

where the amplitude is $L_{0}\left(M, d, T_{s}\right) \simeq 1.05 \times 10^{4}\left(\mathrm{erg} \mathrm{s}^{-1}\right) \frac{M}{M_{\odot}} \frac{d}{\mathrm{~m}} \frac{T_{s}^{4}}{K^{4}}$, and the corrected phase profile $\Phi^{\prime}(e, \theta, \phi, \alpha, t)$ is

$$
\Phi^{\prime}(e, \theta, \phi, \alpha, t)=\Upsilon(e, \alpha) \Phi(\theta, \phi, \alpha, t) .
$$

Provided, the correction function is denotetd by $\Upsilon(e, \alpha):=C_{1}(e, \alpha) / 2 \pi r_{g}, \Phi(\theta, \phi, \alpha, t)$ is the phase profile in case of spherical EH [1]:

$$
\Phi(\theta, \phi, \alpha, t) \equiv \sqrt{1-\sin ^{2} \beta \sin ^{2}(\phi+A)} .
$$

Here the spherical triangle, with the lengths of three sides $\theta=(\widehat{z, s}), \alpha=(\widehat{s, n})$ and $\beta=(\widehat{z, n})$, is solved by the law of cosines

$$
\cos \beta(\theta, \alpha, t)=\cos \theta \cos \alpha+\sin \theta \sin \alpha \cos \Omega t, \quad \cos A(\theta, \alpha, t)=\frac{\cos \alpha-\cos \theta \cos \beta}{\sin \theta \sin \beta} .
$$

Consequently, the phase profile (40) can be recast into the form

$$
\begin{aligned}
& \Phi^{\prime}(e, \theta, \phi, \alpha, t)=\frac{2}{\pi \sqrt{1-e^{2}}} \int_{0}^{\pi / 2} \sqrt{1-e_{1}^{2}(\alpha) \sin ^{2} \gamma} d \gamma \Phi(\theta, \phi, \alpha, t)= \\
& \frac{1}{\sqrt{1-e^{2}}}\left[1-\Sigma_{n=1}^{\infty}\left(\frac{(2 n-1) ! !}{2^{n} n !}\right)^{2} \frac{e_{1}^{2 n}(\alpha)}{2 n-1}\right] \Phi(\theta, \phi, \alpha, t),
\end{aligned}
$$

where $n ! !$ is the double factorial $n ! !=\prod_{i=0}^{l}(n-2 i), l=[n / 2]-1$. The Fig. 5 and Fig. 6 are the examples, revealing the diversity of the behavior of corrected phase profiles versus the time, viewed at given position angles, for different values of eccentricity.

However, at this point we cut short our investigation by noting that there is still very much to be gained by further study of the issues that we have raised in this paper. 


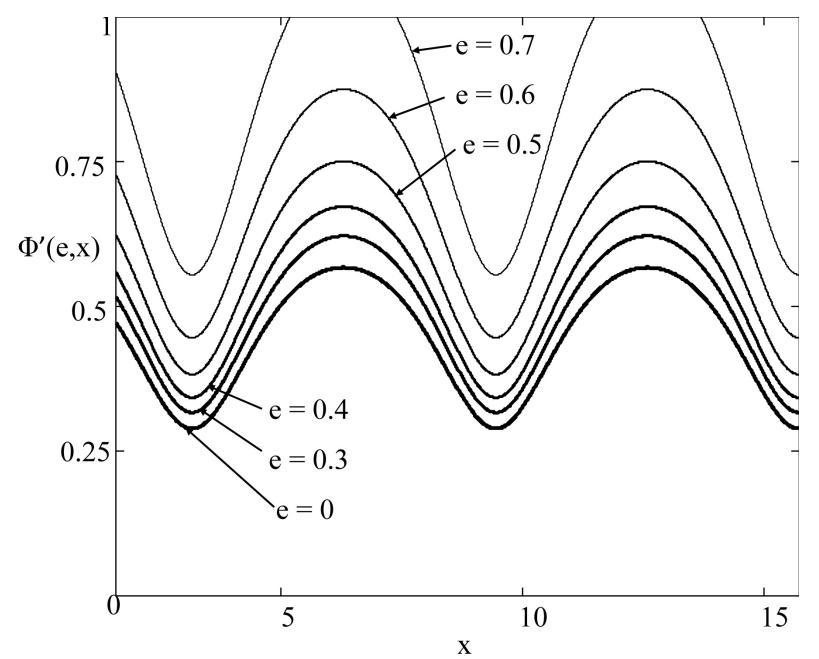

Figure 5. The corrected phase profiles $\Phi^{\prime}(e, x=\Omega t)$ at position angles $\theta=45^{\circ}, \phi=18^{0}, \alpha=90^{\circ}$.

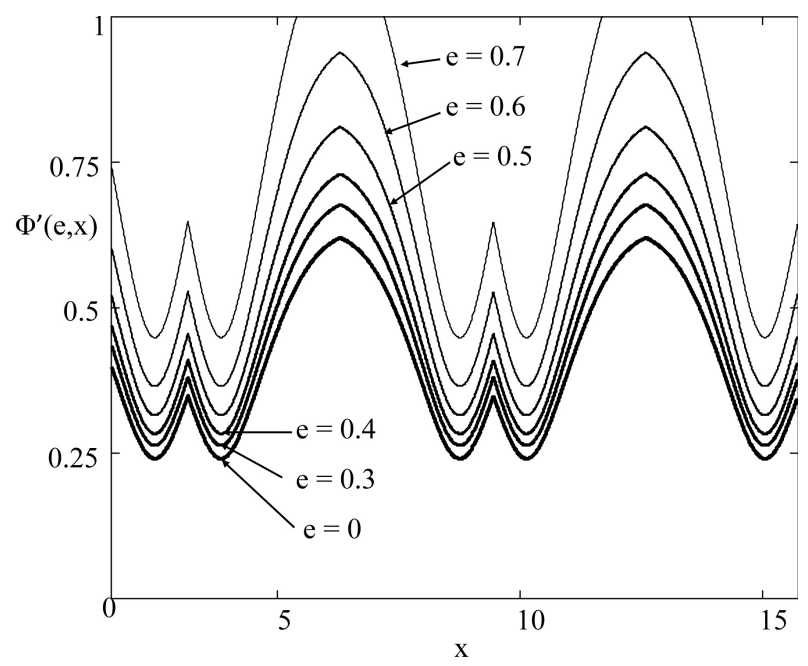

Figure 6. The corrected phase profiles $\Phi^{\prime}(e, x=\Omega t)$ at position angles $\theta=45^{\circ}, \phi=30^{\circ}, \alpha=60^{\circ}$. 


\section{Concluding Remarks}

In this report, we further expose the assertions made in $\mathrm{MTBH}$ via reviewing the microscopic model of stationary and axisymmetric rotating SPC. We supplement our previous investigation [1] of observed unusual high luminosity of NuSTAR X-ray pulsations from M82X-2, further, by more rigorous analytical treatment of rotating SPC. We derive corresponding field equations and obtain both internal and global vacuum spacetime solutions.

There are deep conceptual and technical problems involved, and these provide scope for the arguments discussed, which are carefully presented in both mathematical and physical terms. Exploring spontaneous breaking of gravitation gauge symmetry at huge energies, MTBH has smeared out a central singularity of black hole replacing it by the equilibrium SPC inside event horizon. This ultimately circumvents the principle problem, existing in $\mathrm{PMBH}$, of an observer's inability to access the degrees of freedom that are hidden beyond the horizon, and a necessity to assign the misleading entropy to black hole. The physical entropy of SPC is a measure of the large number of the real thermodynamical microstates, which are compatible with the ergodicity.

The most remarkable feature of microscopic model of a rotating black hole is that, in earlier part of its lifetime, the external physics outside of outer oblate event horizon of accretion onto a black hole is identical to the processes in Kerr's model. But, there is also a crucial difference between internal physics of Kerr and microscopic rotating black hole models, that is, a central ring singularity of the Kerr black hole cannot occur, which is now replaced by finite though unbelievably extreme conditions held in the central part of rotating SPC, where the static observers exist. This has then made room for growth and merging properties of black holes.

The OEH introduces the corrections to the characteristic phase profile of the previous model.

\section{Acknowledgments}

The very helpful comments from the anonymous referee that have essentially improve the quality of the final manuscript are much appreciated.

\section{Appendix A: Underlying Gravitation Theory}

There are some GR plagued conceptual problems, among which are the energy-momentum conservation laws of gravitational interacting fields, the localization of energy of gravitation waves, the role of singularities or black holes, and also severe problems involved in quantum gravity. The standard Riemannian (and its extensions) space interacting quantum field theory cannot be a satisfactory theory, because Riemannian geometry, in general, does not admit a group of isometries, and it is impossible to define energy-momentum as Noether local currents related to exact symmetries. This, in turn, posed severe problem of non-uniqueness of the physical vacuum and the associated Fock space, since the Poincaré transformations no longer act as isometries. The following two key questions should be addressed yet: (i) the absence of the definitive concept of space-like separated points, particularly, in the canonical approach, and the light-cone structure at each spacetime point; (ii) the separation of positive- and negative-frequencies for completeness of the Hilbert-space description. Due to that, a definition of positive frequency modes cannot, in general, be unambiguously fixed in the past and future, which leads to $\mid$ in $>\neq \mid$ out $>$, because the state $\mid$ in $>$ is unstable against decay into many particle $\mid$ out $>$ states due to interaction processes allowed by lack of Poincaré invariance. A non-trivial Bogolubov transformation between past and future positive frequency modes implies that particles are created from the vacuum and this is one of the reasons for $\mid$ in $>\neq \mid$ out $>$.

Keeping in mind as aforesaid, the aim of the appendices B-F is to outline briefly the key points of the underlying MTBH gravitation theory, as a guiding principle to make this report understandable. Discussed gravitational theory is consistent with general relativity for available solar system observational verifications [10], which offers many opportunities to improve tests of relativistic gravity. Moreover, we rather shown that this agreement is satisfactory even up to the limit of neutron stars (see [9] and references therein). 
But the proposed theory manifests its virtues when applied to the physics of internal structure of galactic nuclei. It yields the gravitational interaction at huge energies drastically different from earlier suggested schemes. This theory involves a principle revision of a role of local internal symmetries in physical concept of curved geometry, and that explores the most important processes of spontaneous breaking of gravitation gauge symmetry and rearrangement of vacuum state.

There are several important topics not touched upon here, which will eventually benefit from the proposed theory. Although some key theoretical ideas were introduced with a satisfactory substantiation, we have also attempted to maintain a balance between being overly detailed and overly schematic. Below, we develop, as from first principles, on under the framework of the spacetime deformation theory, the general gauge principle (GGP), and the spontaneous breaking of gravitation gauge symmetry.

\section{Appendix B: A First Glance at Spacetime Deformations}

In the framework of spacetime deformation theory ([19] and references therein), we consider a smooth deformation map $\Omega: M_{4} \rightarrow \widetilde{\mathcal{M}}_{4}$, written in the terms of the world-deformation tensor $\Omega$, the general, $\widetilde{\mathcal{M}}_{4}$, and the flat, $M_{4}$, smooth differential 4 D-manifolds. A following notational conventions will be used throughout this paper. All magnitudes related to the space, $\widetilde{\mathcal{M}}_{4}$, will be denoted with an over ' $\sim$ '. We use the Greek alphabet $(\mu, \nu, \rho, \ldots=0,1,2,3)$ to denote the holonomic world indices related to $\widetilde{\mathcal{M}}_{4}$, and the second half of Latin alphabet $(l, m, k, \ldots=0,1,2,3)$ to denote the world indices related to $M_{4}$. The tensor, $\Omega$, can be written in the form $\Omega=D \psi\left(\Omega_{l}^{m}=D_{\mu}^{m} \psi_{l}^{\mu}\right)$, provided with the invertible distortion matrix $D\left(D_{\mu}^{m}\right)$ and the tensor $\psi\left(\psi_{l}^{\mu} \equiv \partial_{l} \widetilde{x}^{\mu}\right.$ and $\left.\partial_{l}=\partial / \partial x^{l}\right)$.

The principle foundation of a world-deformation tensor comprises the following two steps.

First step. The basis vectors $e_{m}$ at given point $\left(p \in M_{4}\right)$ undergo the distortion transformations by means of the matrix $D$ :

$$
\widetilde{e}_{\mu}=D_{\mu}^{l} e_{l}
$$

Second step. The diffeomorphism $\widetilde{x}^{\mu}(x): M_{4} \rightarrow \widetilde{M}_{4}$ is constructed by seeking new holonomic coordinates $\widetilde{x}^{\mu}(x)$ as the solutions of the first-order partial differential equations:

$$
\widetilde{e}_{\mu} \psi_{l}^{\mu}=\Omega_{l}^{m} e_{m}
$$

where the conditions of integrability, $\partial_{k} \psi_{l}^{\mu}=\partial_{l} \psi_{k}^{\mu}$, and non-degeneracy, $\|\psi\| \neq 0$, necessarily hold $[30,31]$.

For reasons that will become clear in the Appendix $\mathrm{C}$, next we write the norm $d \widetilde{s} \equiv i \widetilde{d}$ of the infinitesimal displacement $d \widetilde{x}^{\mu}$ on the $\widetilde{\mathcal{M}}_{4}$ in terms of the spacetime structures of $M_{4}$ :

$$
i \widetilde{d}=\widetilde{e} \widetilde{\vartheta}=\widetilde{e}_{\mu} \otimes \widetilde{\vartheta}^{\mu}=\Omega_{l}^{m} e_{m} \otimes \vartheta^{l} \in \widetilde{\mathcal{M}}_{4}
$$

The deformation $\Omega: M_{4} \rightarrow \widetilde{\mathcal{M}}_{4}$ comprises the following two subsequent $4 \mathrm{D}$ deformations $\stackrel{\circ}{\Omega}: M_{4} \rightarrow V_{4}$ and $\breve{\Omega}: V_{4} \rightarrow \widetilde{M}_{4}$, where $V_{4}$ is the semi-Riemannian space, $\stackrel{\circ}{\Omega}$ and $\breve{\Omega}$ are the corresponding world deformation tensors. The interested reader is invited to consult the original papers $[19,12,20]$ for further details of the theory of spacetime deformation.

\section{Appendix C: General Gauge Principle (GGP)}

The GGP resolves the problems of GR and Riemannian space interacting quantum field theory mentioned in App.A.

Let us consider the simplest spacetime deformation map, $\Omega: M_{4} \rightarrow V_{4}\left(\breve{\Omega}^{\mu}{ }_{\nu} \equiv \delta_{\nu}^{\mu}\right)$. Accordingly, the quantities denoted by wiggles here refer to $V_{4}$ space, but the quantities referring to flat $M_{4}$ space are left without wiggles as previously. Given the principal fiber bundle $\widetilde{P}\left(V_{4}, G_{V} ; \widetilde{s}\right)$ with the structure group $G_{V}$, the local coordinates $\widetilde{p} \in \widetilde{P}$ are $\widetilde{p}=\left(\widetilde{x}, U_{V}\right)$, where $\widetilde{x} \in V_{4}$ and $U_{V} \in G_{V}$, the total bundle space $\widetilde{P}$ is a smooth manifold, the surjection $\widetilde{s}$ is a smooth map $\widetilde{s}: \widetilde{P} \rightarrow V_{4}$. The collection of matter fields of arbitrary spins $\widetilde{\Phi}(\widetilde{x})$ take values in standard fiber over $\widetilde{x}: \widetilde{s}^{-1}\left(\widetilde{\mathcal{U}}_{i}\right)=\widetilde{\mathcal{U}}_{i} \times \widetilde{F}_{\widetilde{x}}$. The action of 
the structure group $G_{V}$ on $\widetilde{P}$ defines an isomorphism of the Lie algebra $\widetilde{g}$ of $G_{V}$ onto the Lie algebra of vertical vector fields on $\widetilde{P}$ tangent to the fiber at each $\widetilde{p} \in \widetilde{P}$ called fundamental.

Involving a drastic revision of the role of gauge fields in the physical concept of the spacetime deformation, we generalize the standard gauge scheme by exploring a new special type of distortion gauge field. Thereby we also consider the principle fiber bundle, $P\left(M_{4}, U^{l o c} ; s\right)$, with the flat base space $M_{4}$, the structure group $U^{l o c}$ and the surjection $s$. The matter fields $\Phi(x)$ take values in the standard fiber which is the Hilbert vector space where a linear representation $U(x)$ of group $U^{l o c}$ is given. This space can be regarded as the Lie algebra of the group $U^{l o c}$ upon which the Lie algebra acts according to the law of the adjoint representation: $a \leftrightarrow$ ad $a \Phi \rightarrow[a, \Phi]$.

We assume that a distortion massless gauge field $a(x)\left(\equiv a_{n}(x)\right)$ has to act on the external spacetime groups. This field takes values in the Lie algebra of the abelian group $U^{l o c}$. We build up the worlddeformation tensor, $\Omega(F)=D(a) \psi(a)$, where $F$ is the differential form of gauge field $F=(1 / 2) F_{n m} \vartheta^{n} \wedge$ $\vartheta^{m}$.

The two steps (44) and (45), together with the third (47) and fourth (48)- following below, and the Lie group $G_{D}$, are the very foundation of GGP. The gravitation gauge group $G_{V}$ is generated by the hidden local internal symmetry $U^{l o c}$. That is:

Third step. The physical system of the fields $\widetilde{\Phi}(\widetilde{x})$ defined on $V_{4}$ must be invariant under the finite local gauge transformations $U_{V}$ of the Lie group of gravitation $G_{V}$ :

$$
\widetilde{\Phi}^{\prime}(\widetilde{x})=U_{V}(\widetilde{x}) \widetilde{\Phi}(\widetilde{x}), \quad\left[\widetilde{\gamma}^{\mu}(\widetilde{x}) \widetilde{\nabla} \mu \widetilde{\Phi}(\widetilde{x})\right]^{\prime}=U_{V}(\widetilde{x})\left[\widetilde{\gamma}^{\mu}(\widetilde{x}) \widetilde{\nabla} \mu \widetilde{\Phi}(\widetilde{x})\right] .
$$

The invariance of the Lagrangian of matter fields, $L_{\widetilde{\Phi}}$, under the infinite-parameter group of general covariance in $V_{4}$ implies an invariance of $L_{\widetilde{\Phi}}$ under the $G_{V}$ group and vice versa if, and only if, the generalized local gauge transformations of the fields $\widetilde{\Phi}(\widetilde{x})$ and their covariant derivative $\widetilde{\nabla} \widetilde{\Phi}_{(}(\widetilde{x})$ are introduced by finite local $U_{V} \in G_{V}$ gauge transformations (47). The GGP scheme is

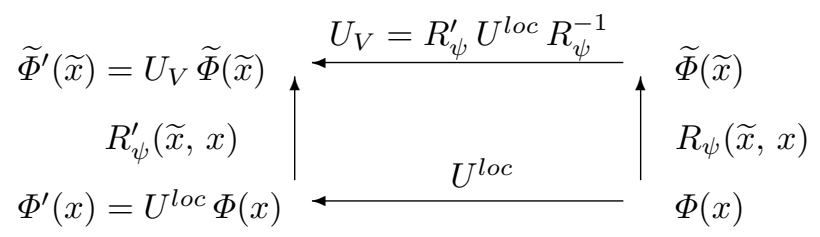

Fourth step. In order for (47) to be always satisfied, a smooth unitary map of all the matter fields and their covariant derivatives holds

$$
R_{\psi}(a): \Phi \rightarrow \widetilde{\Phi}, \quad S(a) R_{\psi}(a):\left(\gamma^{k} D_{k} \Phi\right) \rightarrow\left(\widetilde{\gamma}^{\nu}(\widetilde{x}) \nabla_{\nu} \widetilde{\Phi}\right)
$$

where $R_{\psi}(a)$ (三 $R_{\psi}(\widetilde{x}, x)$ ) is the unitary map matrix, $S(F)$ denotes the gauge invariant scalar function. Determining $R(a)$ and $S(F)$, we may insert first relation in (48) into the second one to obtain the identity, and next equate the coefficients in front of $\Phi$ and $\partial \Phi$ to zero. In the aftermath, we get the two relations to determine $R(a)$ and $S(F)$.

In an illustration of the point at issue, the (48) explicitly reads

$$
\widetilde{\Phi}^{\mu \cdots \delta}(\widetilde{x})=\psi_{l}^{\mu} \cdots \psi_{m}^{\delta} R(a) \Phi^{l \cdots m}(x) \equiv\left(R_{\psi}\right)_{l \cdots m}^{\mu \cdots \delta} \Phi^{l \cdots m}(x),
$$

and that

$$
\widetilde{g}^{\nu}(\widetilde{x}) \nabla_{\nu} \widetilde{\Phi}^{\mu \cdots \delta}(\widetilde{x})=S(F) \psi_{l}^{\mu} \cdots \psi_{m}^{\delta} R(a) \gamma^{k} D_{k} \Phi^{l \cdots m}(x) .
$$

where $S(F) \equiv(1 / 4) \widetilde{\pi}^{-1}(F)=(1 / 4) \psi_{\mu}^{l} D_{l}^{\mu}, \quad D_{k}=\partial_{k}-i \kappa a_{k}$. The $\widetilde{\nabla}_{\mu}$ denotes the covariant derivative agreed with the metric, $\widetilde{g}^{\mu \nu}=(1 / 2)\left(\widetilde{\gamma}^{\mu} \widetilde{\gamma}^{\nu}+\widetilde{\gamma}^{\nu} \widetilde{\gamma}^{\mu}\right): \widetilde{\nabla}_{\mu}=\widetilde{\partial}_{\mu}+\widetilde{\Gamma}_{\mu}$, where $\widetilde{\Gamma}_{\mu}(\widetilde{x})=\frac{1}{2} J^{a b} \widetilde{e}_{a}^{\nu}(\widetilde{x}) \widetilde{\partial}_{\mu} \widetilde{e}_{b \nu}(\widetilde{x})$ is the connection, $J_{a b}$ are the generators of Lorentz group $\Lambda$. The tetrad components $\widetilde{e}_{a}{ }^{\mu}(\widetilde{x})$ are associated with the chosen representation $D(\Lambda)$ by which the $\widetilde{\Phi}(\widetilde{x})$ is transformed as $[D(\Lambda)]_{l \cdots k}^{l^{\prime} \cdots k^{\prime}} \widetilde{\Phi}(\widetilde{x})$, where $D(\Lambda)=$ $I+\frac{1}{2} \widetilde{\omega}^{a b} J_{a b}, \quad \widetilde{\omega}_{a b}=-\widetilde{\omega}_{b a}$ are the parameters of the Lorentz group. One has, for example, to set $\widetilde{\gamma}^{\mu}(\widetilde{x}) \rightarrow$ 
$\widetilde{e}^{\mu}(\widetilde{x})$ for the fields of spin $(j=0,1)$; for vector field $\left[J_{a b}\right]_{k}^{l}=\delta_{a}^{l} \eta_{b k}-\delta_{b}^{l} \eta_{a k}$; but $\widetilde{\gamma}^{\mu}(\widetilde{x})=\widetilde{e}_{a}^{\mu}(\widetilde{x}) \gamma^{a}$ and $J_{a b}=-(1 / 4)\left[\gamma_{a}, \gamma_{b}\right]$ for the spinor field $\left(j=\frac{1}{2}\right)$, where $\gamma^{a}$ are the Dirac matrices.

In the case of zero curvature, one has $\psi_{l}^{\mu}=D_{l}^{\mu}=e^{\mu}{ }_{l}=\left(\partial x^{\mu} / \partial X^{l}\right), \quad\|D\| \neq 0$, where $X^{l}$ are the inertial coordinates. In this way, the conventional gauge theory given on the $M_{4}$ is restored in both curvilinear and inertial coordinates. Although the distortion gauge field $\left(a_{A}\right)$ is a vector field, nevertheless only the gravitational attraction is presented in the proposed theory of gravitation (see $(70)$ ).

\section{Appendix D: Lie Group $G_{D}$ of the Distortion}

We connect the structure group $G_{V}$, further, to nonlinear realization of the Lie group $G_{D}$ of the distortion of extended space $M_{6}\left(\rightarrow \widetilde{M}_{6}\right)$, underlying the $M_{4}$. This extension appears to be indispensable for such a realization. Thereby the space $M_{6}$ reads

$$
M_{6}=R_{+}^{3} \oplus R_{-}^{3}=R^{3} \oplus T^{3}, \quad \operatorname{sgn}\left(R^{3}\right)=(+++), \quad \operatorname{sgn}\left(T^{3}\right)=(---) .
$$

The $e_{(\lambda \alpha)}=O_{\lambda} \times \sigma_{\alpha} \quad(\lambda= \pm, \alpha=1,2,3)$ are linearly independent unit basis vectors at the point (p) of interest of the given three-dimensional space $R_{\lambda}^{3}$. The unit vectors $O_{\lambda}$ and $\sigma_{\alpha}$ imply

$$
<O_{\lambda}, O_{\tau}>={ }^{*} \delta_{\lambda \tau}, \quad<\sigma_{\alpha}, \sigma_{\beta}>=\delta_{\alpha \beta}
$$

where $\delta_{\alpha \beta}$ is the Kronecker symbol, and ${ }^{*} \delta_{\lambda \tau}=1-\delta_{\lambda \tau}$. Three spatial $e_{\alpha}=\xi \times \sigma_{\alpha}$ and three temporal $e_{0 \alpha}=$ $\xi_{0} \times \sigma_{\alpha}$ components are the basis vectors, respectively, in spaces $R^{3}$ and $T^{3}$, where $O_{ \pm}=(1 / \sqrt{2})\left(\xi_{0} \pm\right.$ $\xi), \quad \xi_{0}^{2}=-\xi^{2}=1, \quad<\xi_{0}, \xi>=0$. The $3 \mathrm{D}$ space $R_{ \pm}^{3}$ is spanned by the coordinates $\eta_{( \pm \alpha)}$. By using the $6 \mathrm{D}$ language, we will be able to make a necessary reduction to the conventional $4 \mathrm{D}$ space which can be achieved in the following way.

(i) In the case of free flat space $M_{6}$, the subspace $T^{3}$ is isotropic. And in so far it contributes in line element just only by the square of the moduli $t=\left|\mathbf{x}^{0}\right|, \mathbf{x}^{0} \in T^{3}$, then, the reduction $M_{6} \rightarrow M_{4}=R^{3} \oplus T^{1}$ can be readily achieved if we use $t=\left|\mathbf{x}^{0}\right|$ for conventional time.

(ii) In the case of curved space, the reduction $V_{6} \rightarrow V_{4}$ can be achieved if we use the projection $\left(\widetilde{e}_{0}\right)$ of the temporal component $\left(\widetilde{e}_{0 \alpha}\right)$ of basis six-vector $\widetilde{e}\left(\widetilde{e}_{\alpha}, \widetilde{e}_{0 \alpha}\right)$ on the given universal direction $\left(\widetilde{e}_{0 \alpha} \rightarrow \widetilde{e}_{0}\right)$. By this we choose the time coordinate. Actually, the Lagrangian of physical fields defined on $R_{6}$ is a function of scalars such as $A_{(\lambda \alpha)} B^{(\lambda \alpha)}=A_{\alpha} B^{\alpha}+A_{0 \alpha} B^{0 \alpha}$, then upon the reduction of temporal components of six-vectors $A_{0 \alpha} B^{0 \alpha}=A^{0 \alpha}<\widetilde{e}_{0 \alpha}, \widetilde{e}_{0 \beta}>B^{0 \beta}=A^{0}<\widetilde{e}_{0}, \widetilde{e}_{0}>B^{0}=A_{0} B^{0}$ we may fulfill a reduction to $V_{4}$.

A distortion of the basis (52) comprises the following two steps. We, at first, consider distortion transformations of the ingredient unit vectors $O_{\tau}$ under the distortion gauge field $(a)$ :

$$
\widetilde{O}_{(+\alpha)}(a)=\mathcal{Q}_{(+\alpha)}^{\tau}(a) O_{\tau}=O_{+}+\kappa a_{(+\alpha)} O_{-}, \quad \widetilde{O}_{(-\alpha)}(a)=\mathcal{Q}_{(-\alpha)}^{\tau}(a) O_{\tau}=O_{-}+\kappa a_{(-\alpha)} O_{+},
$$

where $\mathcal{Q}\left(=\mathcal{Q}_{(\lambda \alpha)}^{\tau}(a)\right)$ is an element of the group $Q$. This violates the first relation in (52) because of $\widetilde{O}_{(\lambda \alpha)}^{2}(a)=2 \_a_{(\lambda \alpha)} \neq 0$ for given $\lambda$ and $\alpha$.

The (53), in turn, induces the distortion transformations of the ingredient unit vectors $\sigma_{\beta}$, which undergo the rotations, $\widetilde{\sigma}_{(\lambda \alpha)}(\theta)=\mathcal{R}_{(\lambda \alpha)}^{\beta}(\theta) \sigma_{\beta}$. The $\mathcal{R}(\theta) \in S O(3)$ is the element of the group of rotations of planes involving two arbitrary axes around the orthogonal third axis in the given ingredient space $R_{\lambda}^{3}$. Then, the resulting basis vectors $\widetilde{\sigma}_{(\lambda \alpha)}(\theta)$ of each three-dimensional ingredient space $R_{\lambda}^{3}$ retain the orthogonality condition between themselves, but violate it with respect to the basis vectors of different ingredient spaces. That is, $<\widetilde{\sigma}_{(\lambda \alpha)}, \widetilde{\sigma}_{(\tau \beta)}>_{\alpha \neq \beta} \neq 0$, at $\lambda \neq \tau$. In fact, distortion transformations of basis vectors $(O)$ and $(\sigma)$ are not independent, but rather are governed by the spontaneous breaking of the distortion symmetry. To avoid a further proliferation of indices, thenceforth we will use upper case Latin $(A)$ in indexing $(\lambda \alpha) \quad(\lambda= \pm ; \alpha=1,2,3)$, etc.

The infinitesimal transformations read

$$
\delta \mathcal{Q}_{A}^{\tau}(a)=\kappa \delta a_{A} X_{\lambda}^{\tau} \in Q, \quad \delta \mathcal{R}(\theta)=-\frac{i}{2} M_{\alpha \beta} \delta \omega^{\alpha \beta} \in S O(3)
$$


provided $X_{\lambda}^{\tau}={ }^{*} \delta_{\lambda}^{\tau}$ and $I_{i}=\frac{\sigma_{i}}{2}$, where $\sigma_{i}$ are the Pauli matrices, $M_{\alpha \beta}=\varepsilon_{\alpha \beta \gamma} I_{\gamma}$ and $\delta \omega^{\alpha \beta}=\varepsilon_{\alpha \beta \gamma} \delta \theta_{\gamma}$. The transformation matrix $D(a, \theta)=\mathcal{Q}(a) \times \mathcal{R}(\theta)$ is an element of the distortion group $G_{D}=Q \times S O(3)$ :

$$
D_{\left(d a^{A}, d \theta^{A}\right)}=I+d D_{\left(a^{A}, \theta^{A}\right)}, \quad d D_{\left(a^{A}, \theta^{A}\right)}=i\left[d a^{A} X_{A}+d \theta^{A} I_{A}\right],
$$

where $I_{A} \equiv I_{\alpha}$ at given $\lambda$.

The nonlinear realization technique or the method of phenomenological Lagrangians (see [10] and references therein) provides a way to determine the transformation properties of fields defined on the quotient space. We treat the distortion group $G_{D}$ and its stationary subgroup $H=S O(3)$, respectively, as the dynamical group and its algebraic subgroup. The generators $X_{A}(54)$ of the group $Q$ do not complete the group $H$ to the dynamical group $G_{D}$, therefore, they cannot be interpreted as the generators of the quotien space $G_{D} / H$, and the distortion fields $a_{A}$ cannot be identified directly with the Goldstone fields arisen in spontaneous breaking of the distortion symmetry $G_{D}$. These objections, however, can be circumvented, because, as it is shown by [10], the distortion group $G_{D}=Q \times S O(3)$ can be mapped in a one-to-one manner onto the group $G_{D}=S O(3) \times S O(3)$, which is isomorphic to the chiral group $S U(2) \times S U(2)$. The method of phenomenological Lagrangians is well known for this group. Hence we arrive at

$$
\tan \theta_{A}=-\kappa a_{A} .
$$

Given the distortion field $a_{A}$, the key relation (56) uniquely determines six angles $\theta_{A}$ of rotations around each of six $(A)$ axes.

The fundamental field is distortion gauge field (a) and, thus, all the fundamental gravitational structures in fact - the metric as much as the coframes and connections - acquire a distortion-gauge induced theoretical interpretation. We study the geometrical structure of the space of parameters in terms of Cartan's calculus of exterior forms and derive the Maurer-Cartan structure equations, where the distortion fields (a) are treated as the Goldstone fields [10].

\section{Appendix E: Spontaneous Breaking of Gravitation Gauge Symmetry}

In the realization of the group $G_{V}$ we implement the abelian local group [10],

$$
U^{\text {loc }}=U(1)_{Y} \times \bar{U}(1) \equiv U(1)_{Y} \times \operatorname{diag}[S U(2)]
$$

on the space $M_{6}(51)$ (spanned by the coordinates $\eta$ ), with the group elements of $\exp \left[i \frac{Y}{2} \theta_{Y}(\eta)\right]$ of $U(1)_{Y}$ and $\exp \left[i T^{3} \theta_{3}(\eta)\right]$ of $\bar{U}(1)$. This group leads to the renormalizable theory, because gauge invariance gives a conservation of charge, and it also ensures the cancelation of quantum corrections that would otherwise result in infinitely large amplitudes. This has two generators, the third component $T^{3}$ of isospin $\boldsymbol{T}$ related to the Pauli spin matrix $\frac{\tau}{2}$, and hypercharge $Y$ implying $Q^{d}=T^{3}+\frac{Y}{2}$, where $Q^{d}$ is the distortion charge operator assigning the number -1 to particles, but +1 to anti-particles. The group (57) entails two neutral gauge bosons of $\bar{U}(1)$, or that coupled to $T^{3}$, and of $U(1)_{Y}$, or that coupled to the hypercharge $Y$.

Spontaneous symmetry breaking can be achieved by introducing the neutral complex scalar Higgs field. Minimization of the vacuum energy fixes the non-vanishing vacuum expectation value (VEV), which spontaneously breaks the theory, leaving the $U(1)_{d}$ subgroup intact, i.e. leaving one Goldstone boson. The left Goldstone boson is gauged away from the scalar sector, but it essentially reappears in the gauge sector providing the longitudinally polarized spin state of one of gauge bosons that acquires mass through its coupling to Higgs scalar. The two neutral gauge bosons were mixed to form two physical orthogonal states of the massless component of distortion field (71) $\left(a:=a_{(\lambda \alpha)}\right.$, with $\left.M_{a}=0\right)$, which is responsible for gravitational interactions, and its massive component $(72)\left(\bar{a}:=\bar{a}_{(\tau \beta)}\right.$, with $\left.M_{\bar{a}} \neq 0\right)$, which is responsible for the ID-regime.

Hence, a substantial change of the properties of the spacetime continuum besides the curvature may arise at huge energies. The theory is renormalizable, because gauge invariance gives conservation of charge, also ensures the cancelation of quantum corrections that would otherwise result in infinitely large amplitudes. Without careful thought we expect that in this framework the renormalizability of the theory will not be spoiled in curved space-time too, because, the infinities arise from ultra-violet properties of Feynman integrals in momentum space which, in coordinate space, are short distance properties, and locally (over short distances) all curved space-times look like maximally symmetric (flat) space. 


\section{Appendix F: Field Equations}

In pursuing our goal further, we are necessarily led to extend a whole framework of GGP to $12 \mathrm{D}$ smooth differentiable manifold:

$$
M_{12}=M_{6} \oplus \bar{M}_{6} .
$$

The $M_{6}$ relates to the spacetime (51), but the $\bar{M}_{6}$ is displayed as a space of inner degrees of freedom. The

$$
e_{(\lambda, \mu, \alpha)}=O_{\lambda, \mu} \otimes \sigma_{\alpha} \quad(\lambda, \mu=1,2 ; \alpha=1,2,3)
$$

are basis vectors at the point $p(\zeta)$ of $M_{12}$ :

$$
<O_{\lambda, \mu}, O_{\tau, \nu}>={ }^{*} \delta_{\lambda, \tau}{ }^{*} \delta_{\mu, \nu}, \quad O_{\lambda, \mu}=O_{\lambda} \otimes O_{\mu}, \quad O_{\lambda, \mu} \leftrightarrow{ }^{*} R^{4}={ }^{*} R^{2} \otimes{ }^{*} R^{2}, \quad \sigma_{\alpha} \leftrightarrow R^{3},
$$

where $\zeta=(\eta, u) \in M_{12},\left(\eta \in M_{6}\right.$ and $\left.u \in \bar{M}_{6}\right)$. So, the decomposition (51), together with

$$
\bar{M}_{6}=\bar{R}_{+}^{3} \oplus \bar{R}_{-}^{3}=\bar{T}^{3} \oplus \bar{P}^{3}, \quad \operatorname{sgn}\left(\bar{T}^{3}\right)=(+++), \quad \operatorname{sgn}\left(\bar{P}^{3}\right)=(---),
$$

holds.

The 12-dimensional basis $(e)$ transforms under the distortion gauge field $a(\zeta)\left(\zeta \in M_{12}\right)$ :

$$
\widetilde{e}=D(a) e
$$

where the distortion matrix $D(a)$ reads $D(a)=C(a) \otimes R(a)$, provided

$$
\widetilde{O}=C(a) O, \quad \widetilde{\sigma}=R(a) \sigma .
$$

The matrices $C(a)$ generate the group of distortion transformations of bi-pseudo-vectors:

$$
C_{(\lambda \mu \alpha)}^{\tau, \nu}(a)=\delta_{\lambda}^{\tau} \delta_{\mu}^{\nu}+\kappa a_{(\lambda, \mu, \alpha)} \delta_{\lambda}^{\tau *} \delta_{\mu}^{\nu},
$$

but $R(a) \in S O(3)_{\lambda \mu}$-the group of ordinary rotations of the planes involving two arbitrary basis of the spaces $R_{\lambda \mu}^{3}$ around the orthogonal third axes. The angles of rotations are determined according to (56), but now we use the indices $A=(\lambda, \mu, \alpha)$ etc.

The extended field equations follow at once in terms of Euler-Lagrange variations respectively on the $M_{12}$ and $\widetilde{M}_{12}[10]$ :

$$
S=S_{a}+S_{\widetilde{\Phi}}=\int \sqrt{-\eta} L_{a} d^{12} \zeta+\int \sqrt{-g} L_{\widetilde{\Phi}} d^{12} \widetilde{\zeta}
$$

where $L_{a}$ is the Lagrangian of distortion field $(a), L_{\widetilde{\Phi}}$ is the Lagrangian of matter fields. Whereas the dependence on the distortion gauge field comes only through the components of the metrical tensor. The $L_{a}$ is invariant under Lorentz $(\Lambda)$ and $U^{l o c}$ gauge groups. The Lagrangian $L_{\widetilde{\Phi}}$, in turn, is invariant under the gauge group of gravitation, $G_{R}$.

In terms of the Euler-Lagrange variations in $M_{12}$ and $\widetilde{M}_{12}$, we readily obtain

$$
\frac{\delta\left(\sqrt{-\eta} L_{a}\right)}{\delta a_{A}}=-\frac{\partial g^{B C}}{\partial a_{A}} \frac{\delta\left(\sqrt{-g} L_{\widetilde{\Phi}}\right)}{\delta g^{B C}}=-\frac{\sqrt{-g}}{2} \frac{\partial g^{B C}}{\partial a_{A}} \widetilde{T}_{B C}, \quad \frac{\delta \widetilde{L} \widetilde{\Phi}}{\delta \widetilde{\Phi}}=0, \quad \frac{\delta L_{\widetilde{\Phi}}}{\delta \widetilde{\Phi}}=0,
$$

where $\widetilde{T}_{B C}$ is the energy-momentum tensor of the matter fields $\widetilde{\Phi}(\widetilde{\zeta})$. The Lagrangian of distortion gauge field $a_{A}=\left(a_{(\lambda \alpha)}, \bar{a}_{(\tau \beta)}\right)$ defined on the flat space, is the undegenerated Killing form on the Lie algebra of the group $U^{l o c}$ in the adjoint representation

$$
L_{\bar{a}}(\eta)=L_{a}(\eta)=-\frac{1}{4}<F_{A B}(a), F^{A B}(a)>_{K},
$$

which yields

$$
\partial^{B} \partial_{B} a_{A}-\left(1-\zeta_{0}^{-1}\right) \partial_{A} \partial^{B} a_{B}=J_{A}=-\frac{1}{2} \sqrt{g} \frac{\partial g^{B C}}{\partial a_{A}} \widetilde{T}_{B C},
$$

where $F_{A B}(a)$ is the antisymmetrical tensor of gauge field $(a), \zeta_{0}$ is the gauge fixing parameter. 
To render our discussion here more transparent, below we clarify the relation between gravitational and coupling constants. To assist in obtaining actual solutions from the field equations, we may consider the weak-field limit and shall envisage that the right hand side of (68) should be in the form

$$
-\frac{1}{2}\left(4 \pi G_{N}\right) \sqrt{g(x)} \frac{\partial g^{B C}(x)}{\partial x_{A}} \widetilde{T}_{B C} .
$$

Hence, we may assign to the Newton's gravitational constant $G_{N}$ the value

$$
G_{N}=\kappa^{2} / 4 \pi
$$

The curvature of manifold $M_{6} \rightarrow V_{6}$ is the familiar distortion induced by the field components

$$
a_{(1,1, \alpha)}=a_{(2,1, \alpha)} \equiv \frac{1}{\sqrt{2}} a_{(+\alpha)}, \quad a_{(1,2, \alpha)}=a_{(2,2, \alpha)} \equiv \frac{1}{\sqrt{2}} a_{(-\alpha)} .
$$

The other regime of ID is generated by the field components

$$
a_{(1,1, \alpha)}=-a_{(2,1, \alpha)} \equiv \frac{1}{\sqrt{2}} \bar{a}_{(+\alpha)}, \quad a_{(1,2, \alpha)}=-a_{(2,2, \alpha)} \equiv \frac{1}{\sqrt{2}} \bar{a}_{(-\alpha)} .
$$

\section{References}

1. G. Ter-Kazarian, "On the Physical Nature of the Source of Ultraluminous X-ray Pulsations", Astrophys. E Space Sci., vol. 361, issue 1, pp.20, DOI 10.1007/s10509-015-2604-0, 2016.

2. M. Bachetti et al. (24 authors), "An ultraluminous X-ray source powered by an accreting neutron star", Nature, vol. 514, Issue 7521, pp. 202-204, 2014.

3. G. Wiktorowicz, M. Sobolewska, A. Sadowski, K. Belczynski, "Nature of the Extreme Ultraluminous X-Ray Sources", Astrophysical Journal, vol. 810, Issue 1, article id. 20, 8 pp., 2015.

4. A.A. Mushtukov, V.F. Suleimanov, S.S. Tsygankov, J. Poutanen, "On the maximum accretion luminosity of magnetized neutron stars: connecting X-ray pulsars and ultraluminous X-ray sources", MNRAS, vol. 454, Issue 3, pp.2539-2548, 2015.

5. D. Simone; P. Rosalba, S. Luigi, "NuSTAR J095551+6940.8: a highly magnetized neutron star with superEddington mass accretion", MNRAS, vol. 449, Issue 2, pp.2144-2150, 2015.

6. Y.Y. Pan, L.M. Song, C. M. Zhang, H. Tong, "The magnetic ïñAeld evolution of ULX NuSTAR J095551+6940.8 in M82âĂŤa legacy of accreting magnetar", [astro-ph.HE]/1510.08597], 2016.

7. D. Simone, P. Rosalba; P. Alessandro; B. Enrico; S. Luigi, "The accretion regimes of a highly magnetized NS: the unique case of NuSTAR J095551+6940.8", MNRAS, vol. 457, Issue 3, pp.3076-3083, 2016.

8. A. King, J.-P. Lasota, "ULXs: Neutron stars versus black holes", MNRAS Lett., vol. 458, Issue 1, pp.L10-L13, 2016

9. G.T. Ter-Kazarian, "Protomatter and EHE C.R.", J. Phys. Soc. Jpn., Suppl., B, Vol.70, pp.84-98, 2001.

10. G.T. Ter-Kazarian, "Gravitation and inertia; a rearrangement of vacuum in gravity", Astroph. Es Space Sci., Vol. 327, pp.91- 109 , 2010.

11. G. Ter-Kazarian, "Ultra-high energy neutrino fluxes from supermassive AGN black holes", Astrophys. E Space Sci., Vol. 349, pp 919-938, 2014.

12. G. Ter-Kazarian, "Growth of accreting supermassive black hole seeds and neutrino radiation", J. of Astrophysics, vol. 2015, Article ID 205367, p.1, 2015. http://dx.doi.org/10.1155/2015/205367

13. G. Ter-Kazarian, S. Shidhani \& L. Sargsyan, "Neutrino Radiation of The AGN Black Holes", Astrophys. \&6 Space Sci., Vol.310, pp. 93-110, 2007.

14. A. Castellina, F. Donato, "Astrophysics of Galactic charged cosmic rays", 2012, Invited review, in Vol.5 of Planets, Stars and Stellar Systems Editor-in-chief Oswalt, T.D McLean, I.S.; Bond, H.E.; French, L.; Kalas, P.; Barstow, M.; Gilmore,G.F.; Keel, W. (Eds.) 1st Edition., 2012, 4760 p., Springer, ISBN 978-90-481-8817-8, 2011; arxiv:1110.2981[astro-ph.GA]

15. A. Letessier-Selvon, T. Stanev, "Ultrahigh energy cosmic-rays", Rev. Mod. Phys., Vol. 83, pp. 907-942, 2011; [astro-ph.HE]/1103.0031]

16. G.Sigl, lectures given at ISAPP School "Neutrino Physics and Astrophysics", Villa Monastero, Varenna, Italy, 2011; arxiv:1202.0466[astro-ph.HE]

17. G. Ter-Kazarian, "Spacetime Deformation-Induced Inertia Effects", Advances in Mathematical Physics, vol. 2012, Article ID 692030, p.1, 2012; http://dx.doi.org/10.1155/2012/692030 
18. G.T. Ter-Kazarian, "Gravitation Gauge Group", Nuovo Cimento, Vol.112B, pp.825-838, 1997.

19. G. Ter-Kazarian, "Two-step spacetime deformation-induced dynamical torsion", Class. Quantum Grav., vol. 28, 055003, 2011; arXiv:1102.2491[gr-qc]

20. G. Ter-Kazarian, "Modified Theories of Gravitation behind the Spacetime Deformation", Physics Research International, vol. 2015, Article ID 152846, p.1, 2015; http://dx.doi.org/10.1155/2015/152846

21. P.A.M. Dirac, "Fixation of Coordinates in the Hamiltonian Theory of Gravitation", Phys. Rev., vol. 114, p.924, 1959.

22. P.A.M. Dirac, Lectures on quantum mechanics, Belfer Graduate School of Science Monograph Series. No2, Yeshiva University, New York, 1964.

23. R.Arnowitt, S. Deser \& C.W. Misner, in Recent Developments in General Relativity, Warsaw: Polish Scientific Publishers, 1962.

24. B. Carter, "The commutation property of a stationary, axisymmetric system", Comm. Math. Phys., vol. 17, Issue 3, pp 233-238, 1970.

25. J.M. Bardeen, Astrophys. J., "A Variational Principle for Rotating Stars in General Relativity", Astrophysical Journal, vol. 162, p.71, 1970.

26. S. Bonazzola, E. Gorgoulhon, "A virial identity applied to relativistic stellar models", Class. Quantum Grav., vol. 11, Issue 7, pp. 1775-1784, 1994.

27. P.K. Townsend, "Black holes", Lecture notes, 1997; arxiv:9707012[gr-qc].

28. A. Komar, "Covariant Conservation Laws in General Relativity", Phys.rev., vol. 113, p.934, 1959.

29. D.C. Robinson, "Uniqueness of the Kerr black hole", Phys. Rev. Lett., vol. 34, pp. 905, 906, 1975.

30. L.S. Pontryagin, Continous Groups, Nauka, Moscow, 1984.

31. B.A. Dubrovin et al., Contemporary Geometry, Nauka, Moscow, 1986. 